\title{
Neural Adaptive Tracking Control for an Uncertain Robot Manipulator with Time-Varying Joint Space Constraints
}

\author{
Hamed N. Rahimi ${ }^{\text {a }}$, Ian Howard ${ }^{\mathrm{b}}$, and Lei Cui ${ }^{\mathrm{c}}$ \\ Department of Mechanical Engineering, Curtin University, Perth, 6102, Western Australia \\ a. Email: hamed.rahiminohooji@ postgrad.curtin.edu.au; Tell: (61) 89266 7591; Orcid: 0000-0001-9429-7164 \\ b. Email: i.howard@ curtin.edu.au; Orcid: 0000-0003-3999-9184 \\ c. Email: lei.cui@ curtin.edu.au; Orcid: 0000-0003-2283-5079
}

\begin{abstract}
This paper presents a control design for a robotic manipulator with uncertainties in both actuator dynamics and manipulator dynamics subject to asymmetric time-varying joint space constraints. Tangent-type time-varying barrier Lyapunov functionals (tvBLFs) are constructed to ensure no constraint violation and to remove the need for transforming the original constrained system into an equivalent unconstrained one. Adaptive Neural Networks (NNs) are proposed to handle uncertainties in manipulator dynamics and actuator dynamics in addition to the unknown disturbances. Proper input saturation is employed, and it is proved that under the proposed method the stability and semi-global uniform ultimate boundedness of the closed-loop system can be achieved without violation of constraints. The effectiveness of the theoretical developments is verified through numerical simulations.
\end{abstract}

Keywords: Input saturation; Radial basis function neural networks; Tangent barrier Lyapunov function; Time-varying asymmetric constraints; Uncertain actuator; Uncertain manipulator

\section{Introduction}

Constrained control is becoming increasingly important due to safety issues and performance degradation in the instance of humanoid robots [1, 2], physical human-robot collaboration [3, 4], and assistive robots that guide the motion of the patient's limb in the rehabilitation therapy [5]. In these human-robot interacting tasks, the robotic motions are required to be constrained to avoid the potential of damage to humans. For example, in the rehabilitative robotic arm therapy application, the motion needs to be restricted according to the human partner physical upper-limb dimensions and reaching limits to avoid patient injuries. Therefore, rigorous constraint handling should be carefully managed within the adaptive interactive robotic control.

Numerious techniques for control of the robotic systems have been developed to accommodate various forms of constraints. Some are based on adaptive position/force control [6], adaptive vision and force tracking control [7] or impedance control [8]. In addition, several researchers developed unconventional methods to handle constraints in robotic control. For example, danger field quantity was introduced in [9] for safety-oriented control and danger assessment of robotic manipulators, and the distributed distance sensor approach was proposed in [10] to improve human safety in industrial environments by assessing the level of danger induced by the robot. Also, error transformation technique was used to asymptotic tracking controller design for uncertain robotic systems with external disturbances and time- 
varying constraints on the system state [11].

Motion planning has also been extensively studied to deal with robot constraint avoidance [12, 13 ]. Potential field method was developed to deal with the robot safety issue on the path planning and the real-time control [14]. The quadratic programming based optimal control method was developed for redundant robot manipulators with variable joint-velocity constraints [15]. Optimal motion planning was proposed for mobile robots in static and dynamic obstructed environments combining open-loop optimal control and the potential field method $[16,17]$. However, the trajectory in online optimization methods has to be calculated for various situations, which significantly increases the computational burden. In addition, these methods typically suffer from the implementation of the control inputs at the kinematic level, resulting in them not being able to cope with the dynamic uncertainties.

Barrier Lyapunov Functions (BLFs) have been developed to bound and suppress the propagation of system error [18-20]. Different from the conventional Lyapunov functions, BLFs escape to infinity when associated limits are exceeded. Hence, bounding the BLFs in closed loop systems can prevent violation of constraints along the system trajectories [21]. In addition, as the BLFs control design is constructive based on the direct method of Lyapunov, its computational burden is significantly reduced compared with online motion planning and optimization methods [22]. Different types of BLFs are exploited like logarithm BLFs [20], integral BLFs [23], tangent BLFs [24, 25], secant BLFs [26], and cotangent BLFs [27]. The BLFs based control has been utilized to handle several practical systems with constraints like direct current (DC) motors [28, 29], wind turbines [30], flexible structure systems [31-34], and aerial vehicles [19, 35-37]. Logarithm BLF is employed to solve the trajectory tracking control problem of a fully actuated surface vessel with asymmetric output and input constraints [38]. In addition, this method is used for leader-follower formation control for a group of underactuated surface vessels subject to asymmetric range and bearing constraints [39].

The BLFs based control has been employed for constrained control of robotic manipulators. In [40, 41], task space constraints were handled by considering the linearly-in-parameter conditions in robot dynamics. However, when the robot inverse Jacobian matrix is non-linear, e. g. in the case where the kinematics of the robot manipulator is uncertain [42], the linearly-in-parameter conditions do not hold. To solve the problem, [43, 44], and [45] applied BLFs to the tracking control of robot manipulators with output and full state constraints. However, in these studies, only the static bounds for upper and lower constraints were considered while most practical robotic systems are subject to time-varying constraints. In addition, using the BLFs based control, the input control signals would approach infinity as the states approach their constraint limits. This means that the input control signals are not bounded. These problems were tackled in [25], which developed input and state constrained control using tangent-type time-varying BLFs for MIMO systems and verified the method via a two-link robot manipulator. However, the saturated type input constraint with sharp corners was used, which may prevent the backstepping technique to be applied directly [46]. In addition, this study only assumed the upper constraints to bind the states and errors, which is not an appropriate assumption for most practical applications. Furthermore, in all the works mentioned above, the dynamics of the joint actuator was neglected in spite of the actuator dynamics being a significant part of the real robot dynamics. More recently, BLFs were used to address actuator dynamics in control of robot manipulators in the constrained task space [47] and joint space [23]. However, both works were restricted to static 
constraints and unbounded inputs.

On the other hand, generally, NNs [48] and the fuzzy logic [49] have been widely incorporated into adaptive controller design to account for uncertainties in different mechanical systems like wind turbines [50], DC motors [51], unmanned vehicles [52], underwater vehicles [53], and marine vehicles [54]. Due to their outstanding approximation abilities, such methods afford robust and efficient frameworks to accommodate uncertainty and imprecision [55]. Accordingly, adaptive neural [11, 56] or fuzzy [57] control schemes have been developed to address the stability problem of the unknown robotic systems. In addition, reviewing recent literature on adaptive control outlined the interest of using radial basis functions NNs among robotic researchers [58]. This method has a simple and fixed three-layer (input, hidden, and output) architecture. The output linearly combines neuron parameters with the radial basis function of the inputs [59]. Such networks are easy to design and train and compared to other methods in the literature, this approximation approach forms a composite adaptation law in terms of the tracking error and a model prediction error [60]. Furthermore, enjoying advantages of having strong tolerance to input noise, and the ability of online learning, this method has been extensively employed in control of robotic systems [26, 61-64].

Motivated by the aforementioned considerations, in this paper, asymmetric tangent tvBLFs are developed to prevent the joint space constraint violation in control of robotic systems. Both manipulator dynamics and actuator dynamics uncertainties are considered and radial basis function NNs are employed to approximate the system uncertainties and the unknown disturbances. Also, a proper input saturation is developed to address the tracking problem and to ensure uniform boundedness of the system while all signals in the closed-loop system remain bounded.

Compared with the existing literature, the main advantages of this work are as follows. $i$ ) the proposed tvBLFs can handle both time-varying and asymmetric constraints. Also, by defining a new state constraint in this paper, different initial conditions can be relaxed effectively on the starting values of the movement. By that means, more flexible constraints can be modelled for various practical transitions. $i$ i) a new approach in stability analysis of the closed-loop system using tangent tvBLFs is developed by introducing the Lemma 1. By that means, compared to previous works on tangent BLF like [25, 65-67], the control design procedure required fewer parameters to ensure the prevention of constraint violation. iii) in addition to studying the unknown robotic manipulator dynamics as in [45, 68, 69], unknown actuator dynamics have been considered. Moreover, utilizing NNs unknown disturbances have been incorporated into designing of the controller. Also, the unknown interaction force has been compensated without developing additional estimators.

It also worth noting that compared with the recent works on time-varying constrained control of nonlinear systems in [11, 70-73], this paper directly exploits the constraints on the control design. By that means, unlike $[11,71]$ the approach does not require error transformation. In addition, compared to $[72,73]$ transforming the original constrained system into an equivalent unconstrained one is avoided.

The rest of this paper is organized as follows. Section 2 provides preliminaries and formulates the control problem. Section 3 consists of the control design and stability analysis for unknown robotic systems using tangent tvBLFs where it is shown that the joint space constraints are never violated, and the 
uniform boundedness of the closed-loop system is achieved. Simulations are carried out in Section 4 to illustrate the effectiveness of the proposed control. The concluding remarks and a brief summary of the paper are given in Section 5.

\section{Preliminaries and problem formulation}

\subsection{System description}

Consider a $\boldsymbol{n}$ dimensional serial fully-actuated robotic manipulator [74] as,

$$
M(q) \ddot{q}+C(q, \dot{q}) \dot{q}+G(q)=\tau-d_{r}(t, q, \dot{q})-f(t),
$$

where $\boldsymbol{q}=\left[\boldsymbol{q}_{1}, \ldots, \boldsymbol{q}_{n}\right]^{T}$ represents the generalised coordinate vector which may include revolute and/or prismatic joint variables; $\boldsymbol{M}(\boldsymbol{q}) \in \mathbf{R}^{\boldsymbol{n} \times \boldsymbol{n}}$ denotes the inertia matrix, $\boldsymbol{C}(\boldsymbol{q}, \dot{\boldsymbol{q}}) \in \mathbf{R}^{\boldsymbol{n} \times \boldsymbol{n}}$ denotes the centrifugal and Coriolis forces matrix, $\boldsymbol{G}(\boldsymbol{q}) \in \mathbf{R}^{n}$ denotes the gravitational forces/torques vector; $\tau \in \mathbf{R}^{n}$ is the external force/torque vector, $\boldsymbol{d}_{\boldsymbol{r}}(\boldsymbol{t}, \boldsymbol{q}, \dot{\boldsymbol{q}}) \in \mathbf{R}^{n}$ denotes an external disturbance on the robot manipulator; $\boldsymbol{f}(\boldsymbol{t}) \in \mathbf{R}^{n}$ is the environmental force exerted onto the manipulator.

Property 1 [75]. The inertia matrix $\boldsymbol{M}(\boldsymbol{q})$ is symmetric, and positive definite; also $\dot{\boldsymbol{M}}(\boldsymbol{q})-2 \boldsymbol{C}(\boldsymbol{q}, \dot{\boldsymbol{q}})$ is a skew symmetric matrix.

Assumption 1. The force $\boldsymbol{f}(\boldsymbol{t})$ exerted by the environment or human, is uniformly bounded, i.e., there exists a known constant $\overline{\boldsymbol{f}} \in \mathbf{R}^{+}$, such that $|\boldsymbol{f}(\boldsymbol{t})| \leq \overline{\boldsymbol{f}}, \forall \boldsymbol{t} \in[0, \infty)$. Moreover, it is assumed that the external disturbance $\boldsymbol{d}_{\boldsymbol{r}}$, is bounded by $\left\|\boldsymbol{d}_{\boldsymbol{r}}\right\|<\overline{\boldsymbol{d}}_{\boldsymbol{r}}$ where $\overline{\boldsymbol{d}}_{\boldsymbol{r}}$ is an unknown finite number.

In this study, DC motors are considered to actuate the robotic system. Accordingly, the motor voltage is considered as the control input. The dynamics of the motor are described as [76],

$$
\begin{aligned}
& \tau=\boldsymbol{K}_{N} \boldsymbol{I} \\
& \boldsymbol{L} \dot{\boldsymbol{I}}+\boldsymbol{R} \boldsymbol{I}+\boldsymbol{K}_{e} \dot{\boldsymbol{q}}+\boldsymbol{d}_{\boldsymbol{a}}=\boldsymbol{U}(\boldsymbol{u}),
\end{aligned}
$$

where $\boldsymbol{u} \in \mathbf{R}^{n}$ denotes the armature voltage, $\boldsymbol{I} \in \mathbf{R}^{n}$ represents the armature current, $\boldsymbol{U}(\boldsymbol{u}) \in \mathbf{R}^{n}$ is the vector of saturation limiters to the armature voltage $\boldsymbol{u} ; \boldsymbol{d}_{\boldsymbol{a}} \in \mathbf{R}^{\boldsymbol{n}}$ is the additive disturbance voltage, bounded by $\left\|\boldsymbol{d}_{\boldsymbol{a}}\right\|<\overline{\boldsymbol{d}}_{\boldsymbol{a}}$ with $\overline{\boldsymbol{d}}_{\boldsymbol{a}}$ an unknown finite number; $\boldsymbol{K}_{N} \in \mathbf{R}^{n \times n}$ is a diagonal symmetric and positive definite constant matrix which represents the current-torque electro mechanical conversion, also $\boldsymbol{R}, \boldsymbol{L}, \boldsymbol{K}_{e} \in \mathbf{R}^{n \times n}$ are the diagonal constant positive definite matrices which represent the resistance of armature circuit, inductance of armature circuit, and the motor's voltage constant, respectively. 


\subsection{Problem formulation}

This paper formulates the constrained tracking control problem of robot manipulators as:

Consider the given smooth desired trajectory $\boldsymbol{x}_{d}(t)=\left[\boldsymbol{x}_{d 1}, \ldots, \boldsymbol{x}_{d n}\right]^{T}, i=1, \ldots, n$, and the constrained region $\Omega_{q}=\left\{\boldsymbol{q}_{i} \in \mathbf{R}, \boldsymbol{i}=1, \ldots, \boldsymbol{n} \mid \underline{\boldsymbol{k}}_{o i}(\boldsymbol{t})<\boldsymbol{q}_{i}(\boldsymbol{t})<\overline{\boldsymbol{k}}_{o i}(\boldsymbol{t}), \boldsymbol{t} \geq 0\right\}$ with $\overline{\boldsymbol{k}}_{o i}$ and $\underline{\boldsymbol{k}}_{o i}$ being bounded prespecified functions such that $\overline{\boldsymbol{k}}_{o i}(\boldsymbol{t})>\underline{\boldsymbol{k}}_{o i}(\boldsymbol{t}) \forall \boldsymbol{t} \in \mathbf{R}_{+}$, for the integrated robot dynamics given by (1) and (2), find the input voltage of the actuator $\boldsymbol{u}$ such that the joint positon signal $\boldsymbol{q}(\boldsymbol{t})$ tracks a given desired trajectory $\boldsymbol{x}_{\boldsymbol{d}}$ as closely as possible, i.e., $\lim _{\boldsymbol{t} \rightarrow \infty}\left|\boldsymbol{q}_{i}(\boldsymbol{t})-\boldsymbol{x}_{d i}(\boldsymbol{t})\right|=\boldsymbol{\delta}_{i}$ with $\boldsymbol{\delta}_{i}$ considered as small positive constants, while ensuring the boundedness of the closed-loop system with $q(t)$ never leaving the constrained region $\Omega_{q}$, i.e., $\boldsymbol{q}(\boldsymbol{t}) \in \Omega_{q}, \boldsymbol{t}>0$, provided $\boldsymbol{q}(0) \in \Omega_{q}$.

Remark 1. In this work, the control is able to handle a class of time-varying and asymmetric constraints. This can include as special cases static or symmetric time-varying constraints.

Assumption 2. There exist functions $\overline{\boldsymbol{k}}_{d i}(\boldsymbol{t})$ and $\underline{\boldsymbol{k}}_{d i}(\boldsymbol{t}), \boldsymbol{i}=1, \ldots, \boldsymbol{n}$ satisfying $\overline{\boldsymbol{k}}_{d i}(\boldsymbol{t}) \leq \overline{\boldsymbol{k}}_{\boldsymbol{o} i}(\boldsymbol{t})$ and $\underline{\boldsymbol{k}}_{d i}(\boldsymbol{t}) \geq \underline{\boldsymbol{k}}_{o i}(\boldsymbol{t})$ such that $\underline{\boldsymbol{k}}_{d i}(\boldsymbol{t})<\boldsymbol{x}_{d i}(\boldsymbol{t})<\overline{\boldsymbol{k}}_{d i}(\boldsymbol{t}) \quad \boldsymbol{i}=1, \ldots, \boldsymbol{n}, \forall \boldsymbol{t} \geq 0$. Also, there exist positive constants $\overline{\boldsymbol{K}}_{o i}, \underline{\boldsymbol{K}}_{o i}, \boldsymbol{X}_{d 1 i}$ and $\boldsymbol{X}_{d 2 i}$, such that $\left|\dot{\overline{\boldsymbol{k}}}_{o i}(\boldsymbol{t})\right| \leq \overline{\boldsymbol{K}}_{o i},\left|\dot{\boldsymbol{k}}_{o i}(\boldsymbol{t})\right| \geq \underline{\boldsymbol{K}}_{o i},\left|\dot{\boldsymbol{x}}_{d i}(\boldsymbol{t})\right| \leq \boldsymbol{X}_{d 1 i}$ and $\left|\ddot{\boldsymbol{x}}_{d i}(\boldsymbol{t})\right| \leq \boldsymbol{X}_{d 2 i}$, for $\boldsymbol{i}=1, \ldots, \boldsymbol{n}, \forall \boldsymbol{t}>0$.

Assumption 3. There exist positive constant $\overline{\boldsymbol{k}}_{\boldsymbol{m}}, \underline{\boldsymbol{k}}_{m i}, \overline{\boldsymbol{k}}_{\boldsymbol{n} i}$ and $\underline{\boldsymbol{k}}_{n i}$, such that $\underline{\boldsymbol{k}}_{\boldsymbol{m}}<\boldsymbol{k}_{\boldsymbol{m} i}(\boldsymbol{t})<\overline{\boldsymbol{k}}_{\boldsymbol{m} i}$ and $\underline{\boldsymbol{k}}_{n i}<\boldsymbol{k}_{n i}(\boldsymbol{t})<\overline{\boldsymbol{k}}_{n i}, \boldsymbol{i}=1, \ldots, \boldsymbol{n}, \forall \boldsymbol{t} \geq 0$ where $\boldsymbol{k}_{m i}(\boldsymbol{t})$ and $\boldsymbol{k}_{n i}(\boldsymbol{t})$ are time varying barriers on manipulator joint tracking errors, defined by $\boldsymbol{k}_{n i}(t)=\underline{k}_{o i}(t)-\boldsymbol{x}_{d i}(t)$ and $\boldsymbol{k}_{m i}(t)=\overline{\boldsymbol{k}}_{o i}(t)-\boldsymbol{x}_{d i}(t)$.

Remark 2. A number of lower or upper bounds are defined by Assumptions 2, and 3 and in formulating the control problem. These bounds will be used to develop the control algorithm and stability analysis. Nevertheless, these parameters, although existing, will not be involved in designing the control. Accordingly, actual estimation of them will not be required in setting up and implementing the control scheme.

\subsection{Technical lemmas}

Lemma 1 . The following inequality holds for all $|\xi|<1$ :

$$
\tan \left(\frac{\pi}{2} \xi^{2}\right) \leq \pi \xi^{2} \sec ^{2}\left(\frac{\pi}{2} \xi^{2}\right)
$$

\section{Proof. Let}


$\Theta_{1}(\xi)=\pi \xi^{2} \sec ^{2}\left(\pi \xi^{2} / 2\right)-\tan \left(\pi \xi^{2} / 2\right)$ and $\Theta_{2}(\xi)=\Theta_{1}(\xi) \cos ^{2}\left(\pi \xi^{2} / 2\right) ;$ then $\Theta_{2}(\xi)$ becomes $\Theta_{2}(\xi)=\pi \xi^{2}-\sin \left(\pi \xi^{2} / 2\right) \cos \left(\pi \xi^{2} / 2\right)$. Derivation of $\Theta_{2}(\xi)$ with respect to $\xi$ can be given by $\boldsymbol{d} \Theta_{2}(\xi) / d \boldsymbol{\xi}=\pi \xi\left(2-\cos \left(\pi \xi^{2}\right)\right)$. It is obvious that $\left(d \Theta_{2}(\xi) / d \xi\right)<0$, for $\xi<0, \quad\left(d \Theta_{2}(\xi) / d \xi\right)=0$, for $\boldsymbol{\xi}=0$, and $\left(\boldsymbol{d} \Theta_{2}(\boldsymbol{\xi}) / \boldsymbol{d} \boldsymbol{\xi}\right)>0$, for $\boldsymbol{\xi}>0$. Accordingly, considering $\Theta_{2}(0)=0$ it can be obtained that $\Theta_{2}(\xi) \geq 0$ and furthermore, it proves that $\Theta_{1}(\xi) \geq 0$, and accordingly the right-hand side of the inequality (3) is proved.

This Lemma is developed to be used in stability analysis of the closed-loop system using tangent tvBLFs. Note that using this Lemma, compared to previous tangent BLFs like [25, 66, 67], will require fewer parameters to be considered in the design procedure.

Lemma 2. Let $\boldsymbol{Z}:=\left\{\xi \in \mathbf{R}^{n}|| \xi_{i} \mid<1, \boldsymbol{i}=1, \ldots, \boldsymbol{n}\right\} \subset \mathbf{R}^{n}$. Let $\boldsymbol{N}:=\mathbf{R}^{l} \times \boldsymbol{Z} \subset \mathbf{R}^{l+n}$ be open sets. Define the system,

$$
\dot{\eta}=\boldsymbol{h}(\boldsymbol{t}, \boldsymbol{\eta})
$$

where $\boldsymbol{\eta}:=[\omega, \xi]^{T} \in N$, and $\boldsymbol{h}: \mathbf{R}_{+} \times \boldsymbol{N} \rightarrow \mathbf{R}^{l+\boldsymbol{n}}$ is piecewise continuous in $\boldsymbol{t}$ and locally Lipschitz in $\boldsymbol{\eta}$, uniformly in $\boldsymbol{t}$, on $\mathbf{R}_{+} \times \boldsymbol{N}$. Let $\boldsymbol{Z}_{i}:=\left\{\boldsymbol{\xi}_{i} \in \mathbf{R}|| \xi_{i} \mid<1\right\} \subset \mathbf{R}$ and suppose that there exist functions $\boldsymbol{V}_{\boldsymbol{i}}: \boldsymbol{Z}_{\boldsymbol{i}} \rightarrow \mathbf{R}_{+}, \boldsymbol{i}=1, \ldots, \boldsymbol{n}$, and $\boldsymbol{U}: \mathbf{R}^{l} \rightarrow \mathbf{R}_{+}$continuously differentiable and positive definite in their respective domains, such that,

$$
\begin{aligned}
& V_{i}\left(\xi_{i}\right) \rightarrow \infty \text { as }\left|\xi_{i}\right| \rightarrow 1, i=1, \ldots, n \\
& \gamma_{1}(\|\omega\|) \leq \boldsymbol{U}(\boldsymbol{\omega}) \leq \gamma_{2}(\|\omega\|),
\end{aligned}
$$

where $\gamma_{1}$ and $\gamma_{2}$ are class $\boldsymbol{K}_{\infty}$ functions. Let $\boldsymbol{V}(\boldsymbol{\eta}):=\sum_{i=1}^{n} \boldsymbol{V}_{i}\left(\boldsymbol{\xi}_{i}\right)+\boldsymbol{U}(\boldsymbol{\omega})$, and $\boldsymbol{\xi}_{i}(0)$ belongs to the set $Z_{i}$. If the inequality holds,

$$
\dot{\boldsymbol{V}}=\frac{\partial \boldsymbol{V}}{\partial \boldsymbol{\eta}} \boldsymbol{h} \leq-\boldsymbol{v}_{1} \boldsymbol{V}+\boldsymbol{v}_{2},
$$

in the set $\boldsymbol{\eta} \in \boldsymbol{N}$, where $\boldsymbol{v}_{1}$ and $\boldsymbol{v}_{2}$ are positive constants, then $\boldsymbol{\omega}$ remains bounded and $\boldsymbol{\xi}(\boldsymbol{t})$ remains in the open set $\boldsymbol{Z} \forall \boldsymbol{t} \in[0, \infty)$.

Proof. Please refer to [77].

Note that the above lemma establishes the control performance and constraint satisfaction by using BLFs.

Lemma 3. For any constant $\varsigma>0$ and $\phi \in \mathbf{R}$, the following inequality holds, 


$$
0 \leq|\phi|-\phi \tanh \left(\frac{\phi}{\zeta}\right) \leq \boldsymbol{k}_{p} \varsigma
$$

where $\boldsymbol{k}_{p}=0.2785$.

Proof. Please refer to [78].

From this point onwards, for simplifying notation, the time and state dependence of the system are omitted, provided it would not cause confusion.

\section{Control Design}

In this section, the control procedure is designed for the robot dynamics (1) integrated with the motor dynamics (2) to obtain the following objectives:

1) Track the desired position trajectory without violation of constraints on joint angles;

2) Make the velocity error as small as possible;

3) Make the armature current error as small as possible.

To do this, let $\boldsymbol{x}=\left[\boldsymbol{x}_{1}, \boldsymbol{x}_{2}, \boldsymbol{x}_{3}\right]^{T}, \quad$ where $\quad \boldsymbol{x}_{1}=\boldsymbol{q}=\left[\boldsymbol{q}_{1}, \boldsymbol{q}_{2}, \ldots, \boldsymbol{q}_{n}\right]^{T}, \quad \boldsymbol{x}_{2}=\left[\dot{\boldsymbol{q}}_{1}, \dot{\boldsymbol{q}}_{2}, \ldots, \dot{\boldsymbol{q}}_{n}\right]^{T} \quad$ and $\boldsymbol{x}_{3}=\left[\boldsymbol{I}_{1}, \boldsymbol{I}_{2}, \ldots, \boldsymbol{I}_{n}\right]^{T}$ then, the integrated system dynamics can be expressed as,

$$
\begin{aligned}
& \dot{x}_{1}=\boldsymbol{x}_{2} \\
& \dot{\boldsymbol{x}}_{2}=\boldsymbol{M}^{-1}\left(\boldsymbol{\tau}-\boldsymbol{C} \boldsymbol{x}_{2}-\boldsymbol{G}-\boldsymbol{d}_{\boldsymbol{r}}-\boldsymbol{f}\right) \\
& \dot{\boldsymbol{x}}_{3}=-\boldsymbol{L}^{-1}\left(\boldsymbol{R} \boldsymbol{x}_{3}+\boldsymbol{K}_{\boldsymbol{e}} \boldsymbol{x}_{2}+\boldsymbol{d}_{\boldsymbol{a}}-\boldsymbol{U}(\boldsymbol{u})\right) .
\end{aligned}
$$

Define the error variables as $\boldsymbol{e}_{1}=\left[\boldsymbol{e}_{11}, \boldsymbol{e}_{12}, \ldots, \boldsymbol{e}_{1 n}\right]^{T}=\boldsymbol{x}_{1}-\boldsymbol{x}_{\boldsymbol{d}}, \boldsymbol{e}_{2}=\left[\boldsymbol{e}_{21}, \boldsymbol{e}_{22}, \ldots, \boldsymbol{e}_{2 n}\right]^{T}=\boldsymbol{x}_{2}-\boldsymbol{\alpha}$ and as $\boldsymbol{e}_{3}=\left[\boldsymbol{e}_{31}, \boldsymbol{e}_{32}, \ldots, \boldsymbol{e}_{3 n}\right]^{T}=\boldsymbol{x}_{3}-\mathrm{T}_{d}\left(\tau_{d}\right)$ where $\alpha=\left[\alpha_{1}, \boldsymbol{\alpha}_{2}, \ldots, \boldsymbol{\alpha}_{n}\right]^{T}$ and $\tau_{d}=\left[\tau_{d 1}, \tau_{d 2}, \ldots, \tau_{d n}\right]^{T}$ are stabilizing functions to be designed. The signal $\mathrm{T}_{d} \in \mathbf{R}^{n}$ is the saturation limiter to the signal $\tau_{d}$ and is defined by $\mathrm{T}_{d i}=\tau_{M i} \times \tanh \left(\tau_{d i} / \tau_{M i}\right)$ for $\boldsymbol{i}=1,2, \ldots, \boldsymbol{n}$, with $\tau_{M} \in \mathbf{R}^{n}$ being a known bound of the $\tau_{d}$ [46].

This paper employs the tangent tvBLFs for constrained joint space control design as,

$$
V_{x 1, i}=\frac{\boldsymbol{k}_{b i}^{2}}{\pi} \tan \left(\frac{\pi}{2} \xi_{i}^{2}\right)
$$

where $\boldsymbol{k}_{b i}=\boldsymbol{k}_{m i}$, if $\boldsymbol{e}_{1 i}>0$, otherwise $\boldsymbol{k}_{b i}=\boldsymbol{k}_{n i}$, and the error coordinate $\boldsymbol{\xi}_{i}$ is defined as

$$
\xi_{i}=\mu\left(e_{1 i}\right) \frac{e_{1 i}}{k_{m i}}+\left(1-\mu\left(e_{1 i}\right)\right) \frac{e_{1 i}}{k_{n i}}
$$

with $\boldsymbol{\mu}(\bullet)=1$, if $\bullet>0$, otherwise $\boldsymbol{\mu}(\bullet)=0$. Note that the Lyapunov function in (5) is positive definite and continuously differentiable, also $\boldsymbol{C}^{1}$ in the set $\Omega_{\xi}=\left\{\boldsymbol{\xi}_{i}, \boldsymbol{i}=1, \ldots, \boldsymbol{n}|| \boldsymbol{\xi}_{i}(\boldsymbol{t}) \mid<1, \boldsymbol{t} \geq 0\right\}$. In addition, $\boldsymbol{V}_{x 1, i}$ 
will approach to infinity as $\xi_{i}(t) \rightarrow 1$. It is worth mentioning that, using L'Hospital rule, one can show that $\lim _{\boldsymbol{k}_{b i} \rightarrow \infty} \boldsymbol{k}_{b i}^{2} \tan \left(\boldsymbol{\pi} \boldsymbol{e}_{1 i}{ }^{2} / 2 \boldsymbol{k}_{b i}^{2}\right) / \boldsymbol{\pi}=\boldsymbol{e}_{1 i}^{2} / 2$, thus the BLF presented at (5) can be mathematically considered equivalent to the traditional quadratic Lyapunov function, as $\boldsymbol{k}_{b i}$ is considered as an arbitrarily large finite number. By that means, one can simply replace the presented BLF with the quadratic one when no constraints are required. Note that a conventional logarithm-based BLF like [38, 39, 64, 77], or secant type barrier Lyapunov function [26] will not have such property.

Lemma 4. The condition $\left|\boldsymbol{\xi}_{i}\right|<1$ holds iff $-\boldsymbol{k}_{n i} \leq \boldsymbol{e}_{1 i} \leq \boldsymbol{k}_{\boldsymbol{m} i}$.

Proof. Please refer to [77].

Remark 3. To apply the barriers on the manipulator joint tracking errors, it should be noted that in some applications that may not need time-varying or asymmetric joint space constraints, barriers $\boldsymbol{k}_{\boldsymbol{m}}$ and $\boldsymbol{k}_{\boldsymbol{n}}$ can be modified by using static variables $\boldsymbol{k}_{\boldsymbol{m}}$ and $\boldsymbol{k}_{\boldsymbol{n}}$ in the time-constant case or $\boldsymbol{k}_{\boldsymbol{m}}=\boldsymbol{k}_{\boldsymbol{n}}$ in the symmetric case. It also should be noted that some practical applications may need to enforce transient error boundaries without demanding change to the joint space constraints. This situation can be handled by directly designing $\boldsymbol{k}_{\boldsymbol{m}}$ and $\boldsymbol{k}_{\boldsymbol{n}}$, while omitting $\overline{\boldsymbol{k}}_{\boldsymbol{o}}$ and $\underline{\boldsymbol{k}}_{\boldsymbol{o}}$.

To achieve the first goal of our control design, the stabilizing function $\alpha$ is obtained and the constraints on robot angles $\boldsymbol{x}_{1}$ are addressed. Accordingly, choose a tangent tvBLFs function as,

$$
V_{1}=\sum_{i=1}^{n} V_{x 1, i}
$$

Differentiating (7) with respect to time gives,

$$
\dot{\boldsymbol{V}}_{1}=\sum_{i=1}^{n} \frac{2 \boldsymbol{k}_{b i} \dot{\boldsymbol{k}}_{b i}}{\pi} \tan \left(\frac{\pi}{2} \xi_{i}^{2}\right)+\boldsymbol{k}_{b i}^{2} \xi_{i} \dot{\xi}_{i} \sec ^{2}\left(\frac{\pi}{2} \xi_{i}^{2}\right)
$$

where, $\dot{\xi}_{i}$ is time derivation of $\xi_{i}$ and using (6), it can be rewritten as,

$$
\begin{aligned}
\dot{\xi}_{i} & =\mu\left(\boldsymbol{e}_{1 i}\right) \frac{\dot{\boldsymbol{e}}_{1 i} \boldsymbol{k}_{m i}-\boldsymbol{e}_{1 i} \dot{\boldsymbol{k}}_{m i}}{\boldsymbol{k}_{m i} \boldsymbol{k}_{m i}}+\left(1-\mu\left(\boldsymbol{e}_{1 i}\right)\right) \frac{\dot{\boldsymbol{e}}_{1 i} \boldsymbol{k}_{n i}-\boldsymbol{e}_{1 i} \dot{\boldsymbol{k}}_{n i}}{\boldsymbol{k}_{n i} \boldsymbol{k}_{n i}} \\
& =\mu\left(\boldsymbol{e}_{1 i}\right)\left(\frac{\boldsymbol{e}_{2 i}+\alpha_{i}-\dot{\boldsymbol{x}}_{d i}-\boldsymbol{e}_{1 i} \frac{\dot{\boldsymbol{k}}_{m i}}{\boldsymbol{k}_{m i}}}{\boldsymbol{k}_{m i}}\right)+\left(1-\mu\left(\boldsymbol{e}_{1 i}\right)\right)\left(\frac{\boldsymbol{e}_{2 i}+\alpha_{i}-\dot{\boldsymbol{x}}_{d i}-\boldsymbol{e}_{1 i} \frac{\dot{\boldsymbol{k}}_{n i}}{\boldsymbol{k}_{n i}}}{\boldsymbol{k}_{n i}}\right) .
\end{aligned}
$$

Designing the stabilizing function $\alpha$ can be given as, 


$$
\boldsymbol{\alpha}_{i}=\dot{\boldsymbol{x}}_{d i}-\frac{2 \boldsymbol{k}_{b i} \dot{\boldsymbol{k}}_{b i}}{\pi \boldsymbol{e}_{1 i}} \sin \left(\frac{\pi}{2} \xi_{i}^{2}\right) \cos \left(\frac{\pi}{2} \xi_{i}^{2}\right)-\boldsymbol{k}_{1 i} \boldsymbol{e}_{1 i}+\boldsymbol{e}_{1 i} \frac{\dot{\boldsymbol{k}}_{b i}}{\boldsymbol{k}_{b i}}, \quad \boldsymbol{i}=1,2, \ldots, \boldsymbol{n}
$$

where $\boldsymbol{k}_{1 i}>0$. Note that employing L'Hospital rule, one can see that $\lim _{\boldsymbol{e}_{1 i} \rightarrow 0} \sin \left(\pi \boldsymbol{e}_{1 i}{ }^{2} / 2 \boldsymbol{k}_{b i}^{2}\right) \cos \left(\pi \boldsymbol{e}_{1 i}{ }^{2} / 2 \boldsymbol{k}_{b i}^{2}\right) / \boldsymbol{e}_{1 i} \rightarrow 0$, thus singularity will not occur in (10) because of this term [79]. However, since digital computers cannot evaluate $0 / 0$, the analysis uses the Maclaurin series with the first term to solve the problem. Accordingly, the development considers $\lim _{\boldsymbol{e}_{1 i} \rightarrow 0} \sin \left(\pi \boldsymbol{e}_{1 i}{ }^{2} / 2 \boldsymbol{k}_{b i}^{2}\right) \cos \left(\pi \boldsymbol{e}_{1 i}{ }^{2} / 2 \boldsymbol{k}_{b i}^{2}\right) / \boldsymbol{e}_{1 i}=\lim _{\boldsymbol{e}_{1 i} \rightarrow 0} \sin \left(\pi \boldsymbol{e}_{1 i}{ }^{2} / \boldsymbol{k}_{\boldsymbol{b i}}^{2}\right) / 2 \boldsymbol{e}_{1 i} \approx \pi \boldsymbol{e}_{1 i} / 2 \boldsymbol{k}_{b i}^{2}$, when $\left|\boldsymbol{e}_{1 i}\right|<\boldsymbol{\varepsilon}$ for some small positive $\boldsymbol{\varepsilon}$.

Substituting (9), and (10) into (8) gives,

$$
\dot{\boldsymbol{V}}_{1}=\sum_{i=1}^{n} \boldsymbol{e}_{1 i} \boldsymbol{e}_{2 i} \sec ^{2}\left(\frac{\pi}{2} \boldsymbol{\xi}_{i}^{2}\right)-\sum_{i=1}^{n} \boldsymbol{k}_{1 i} \boldsymbol{k}_{b i}^{2} \boldsymbol{\xi}_{i}^{2} \sec ^{2}\left(\frac{\pi}{2} \boldsymbol{\xi}_{i}^{2}\right)
$$

To achieve the second goal of the control design, the intermediate stabilizing function $\tau_{d}$ is designed to make the joint velocity errors, $\boldsymbol{e}_{2}$, as small as possible. In addition, the coupling term $\sum_{i=1}^{n} \boldsymbol{e}_{1 i} \boldsymbol{e}_{2 i} \sec ^{2}\left(\pi \xi_{i}^{2} / 2\right)$ in (11) will be cancelled in this step.

The augmented Lyapunov candidate functional $V_{2}$ can be chosen as,

$$
V_{2}=V_{1}+\frac{1}{2} e_{2}^{T} M e_{2}
$$

The time derivative of $V_{2}$ is then given by,

$$
\dot{V}_{2}=\dot{V}_{1}+e_{2}^{T} M \dot{e}_{2}+e_{2}{ }^{T} \frac{1}{2} \dot{M} e_{2}
$$

Substituting (4) into (13) leads to,

$$
\dot{\boldsymbol{V}}_{2}=\dot{\boldsymbol{V}}_{1}+\boldsymbol{e}_{2}^{T}\left[\tau-\boldsymbol{C} \boldsymbol{\alpha}-\boldsymbol{G}-\boldsymbol{d}_{\boldsymbol{r}}-\boldsymbol{f}-\boldsymbol{M} \dot{\boldsymbol{\alpha}}+\left(\frac{1}{2} \dot{\boldsymbol{M}}-\boldsymbol{C}\right) \boldsymbol{e}_{2}\right]
$$

Substitution of (11) into (14), and letting $\Delta \tau_{d}=\mathrm{T}_{d}-\tau_{d}$, then employing Property 1, and noting $\boldsymbol{\tau}=\boldsymbol{K}_{\boldsymbol{N}}\left(\boldsymbol{e}_{3}+\mathrm{T}_{\boldsymbol{d}}\right)$ then gives,

$$
\begin{aligned}
\dot{\boldsymbol{V}}_{2}= & \sum_{i=1}^{n} \boldsymbol{e}_{1 i} \boldsymbol{e}_{2 i} \sec ^{2}\left(\frac{\boldsymbol{\pi}}{2} \xi_{i}^{2}\right)-\sum_{i=1}^{n} \boldsymbol{k}_{1 i} \boldsymbol{k}_{b i}^{2} \boldsymbol{\xi}_{i}^{2} \sec ^{2}\left(\frac{\boldsymbol{\pi}}{2} \xi_{i}^{2}\right) \\
& -\boldsymbol{e}_{2}{ }^{\boldsymbol{T}}\left(\boldsymbol{C} \boldsymbol{\alpha}+\boldsymbol{G}+\boldsymbol{d}_{\boldsymbol{r}}+\boldsymbol{f}+\boldsymbol{M} \dot{\boldsymbol{\alpha}}\right)+\boldsymbol{e}_{2}{ }^{\boldsymbol{T}} \boldsymbol{K}_{\boldsymbol{N}}\left(\boldsymbol{e}_{3}+\Delta \boldsymbol{\tau}_{\boldsymbol{d}}+\boldsymbol{\tau}_{\boldsymbol{d}}\right) .
\end{aligned}
$$


Accordingly, as $\boldsymbol{e}_{2}=[0,0, \ldots, 0]^{T}, \quad \dot{\boldsymbol{V}}_{2}=-\sum_{i=1}^{n} \boldsymbol{k}_{1 i} \boldsymbol{k}_{b i}^{2} \xi_{i}^{2} \sec ^{2}\left(\pi \xi_{i}^{2} / 2\right) \leq 0$. Thus, using the Barbalat lemma [80], asymptotic stability of the system is drawn. In case of $\boldsymbol{e}_{2} \neq[0,0, \ldots, 0]^{T}$, the intermediate control law $\tau_{d} \in \mathbf{R}^{n}$ can be designed as,

$$
\tau_{d}=\boldsymbol{K}_{N}^{-1}\left[\tau_{\boldsymbol{l}}-\boldsymbol{k}_{2} \boldsymbol{e}_{2}-\Xi\right]
$$

where, $\boldsymbol{k}_{2}=\operatorname{diag}\left[\boldsymbol{k}_{21}, \ldots, \boldsymbol{k}_{2 n}\right]>0$ are positive constant design parameters, $\Xi=\left[\Xi_{1}, \ldots, \Xi_{n}\right]^{T}$ and is defined as $\Xi_{i}=\boldsymbol{e}_{1 i} \sec ^{2}\left(\pi \xi_{i}^{2} / 2\right), \boldsymbol{i}=1,2, \ldots, \boldsymbol{n}$, and the control signal $\tau_{\boldsymbol{l}}$ is defined as

$$
\tau_{l}=\hat{\boldsymbol{W}}_{1}^{\boldsymbol{T}} \boldsymbol{h}_{1}-\hat{\boldsymbol{D}}_{\boldsymbol{r}} \tanh \left(\frac{\boldsymbol{e}_{2}}{\boldsymbol{\eta}_{1}}\right)-\overline{\boldsymbol{f}} \tanh \left(\frac{\boldsymbol{e}_{2}}{\boldsymbol{\eta}_{2}}\right)
$$

To design the control $\tau_{l}$ in (17) radial basis function NNs are employed to approximate the uncertainties, where $\hat{W}_{1}=\left[\hat{W}_{11}, \ldots, \hat{W}_{1 n}\right]^{T} \in \mathbf{R}^{n \times n}$ is the estimation of ideal weight $\boldsymbol{W}_{1}^{*} \in \mathbf{R}^{n \times n}$ of the NNs. Also, in view of the NNs explanation [81], the term $\boldsymbol{C} \boldsymbol{\alpha}+\boldsymbol{G}+\boldsymbol{M} \dot{\boldsymbol{\alpha}}-\boldsymbol{K}_{N} \Delta \boldsymbol{\tau}_{\boldsymbol{d}}=\boldsymbol{W}_{1}^{* T} \boldsymbol{h}_{1}\left(\boldsymbol{Z}_{1}\right)+\boldsymbol{\varepsilon}_{1}$ is defined, where $\varepsilon_{1}$ is bounded as $\left|\varepsilon_{1}\right| \leq \bar{\varepsilon}_{1}$ with $\bar{\varepsilon}_{1}>0$ being an unknown constant; $Z_{1} \in \mathbf{R}^{n \times 3}$ is an input vector and expressed as $Z_{1}=\left[\boldsymbol{x}_{1}{ }^{T}, \boldsymbol{x}_{2}{ }^{T}, \boldsymbol{\alpha}^{T}\right] ; \boldsymbol{h}_{1}\left(\boldsymbol{Z}_{1}\right)=\left[\boldsymbol{h}_{11}\left(\boldsymbol{Z}_{1}\right), \boldsymbol{h}_{12}\left(\boldsymbol{Z}_{1}\right), \ldots, \boldsymbol{h}_{1 l}\left(\boldsymbol{Z}_{1}\right)\right]^{T}$ is a basis function vector with $\boldsymbol{h}_{1 i}\left(Z_{1}\right)$ for $\boldsymbol{i}=1, \ldots, l$, being the Gaussian functions and defined by $\boldsymbol{h}_{1 i}\left(\boldsymbol{Z}_{1}\right)=\exp \left(-\left(\boldsymbol{Z}_{1}-\boldsymbol{\vartheta}_{i}\right)^{\boldsymbol{T}}\left(\boldsymbol{Z}_{1}-\boldsymbol{\vartheta}_{i}\right) / \boldsymbol{\psi}_{i}^{2}\right)$ with $\boldsymbol{\vartheta}_{i}=\left[\boldsymbol{\vartheta}_{i 1}, \boldsymbol{\vartheta}_{i 2}, \ldots, \boldsymbol{\vartheta}_{i m}\right]^{T}$ being the center of the $\boldsymbol{i}^{\boldsymbol{t}}$ NNs input element, and $\psi_{i}$ being the width of the Gaussian functions. $\hat{\boldsymbol{D}}_{\boldsymbol{r}} \in \mathbf{R}^{n}$ is the estimations of unknown finite numbers $\boldsymbol{D}_{\boldsymbol{r}} \in \mathbf{R}^{\boldsymbol{n}}$ where $\left|\boldsymbol{\varepsilon}_{1 i}+\overline{\boldsymbol{d}}_{\boldsymbol{r} i}\right|<\boldsymbol{D}_{\boldsymbol{r} i}$ for $\boldsymbol{i}=1, \ldots, \boldsymbol{n} ; \boldsymbol{\eta}_{1}>0, \boldsymbol{\eta}_{2}>0$ are small positive numbers.

Remark 4. The control signal (17) consists of three parts:

1) The first term, $\hat{\boldsymbol{W}}_{1}^{T} \boldsymbol{h}_{1}$, is designed to approximate the unknown nonlinear robotic manipulator dynamics, and input difference $\Delta \tau_{d}$. It uses the radial basis function NNs for the approximation and adapts online using the first adaptive law in (24).

2) The second term, $-\hat{\boldsymbol{D}}_{\boldsymbol{r}} \tanh \left(\boldsymbol{e}_{2} / \boldsymbol{\eta}_{1}\right)$, is designed to cope with the external disturbance, and approximation errors arisen from the NN approximation. It deals with the system with unknown bounds employing the 3 , and using the third adaptive law given by (24).

3) The third term, $-\overline{\boldsymbol{f}} \tanh \left(\boldsymbol{e}_{2} / \boldsymbol{\eta}_{2}\right)$, is included to handle the unknown environmental force $\boldsymbol{f}$. Note that since the bound on $f$ is assumed to be known, Lemma 3 can be used to cope with the problem without the need of developing a new adaptive law. 
Remark 5. As the joint positions approach to their boundaries, the value of control $\tau_{d}$ in (16) would increase remarkably since as $\left|\xi_{i}\right| \rightarrow 1$, then $\sec ^{2}\left(\pi \xi_{i}^{2} / 2\right)=\sin ^{2}\left(\pi \xi_{i}^{2} / 2\right) / \cos ^{2}\left(\pi \xi_{i}^{2} / 2\right) \rightarrow \infty$. This may be a source of performance degradation, and may cause dangerous conditions in real applications. This paper handles the problem by designing the input saturation so that it can improve the system reliability, and ensure the safety in operation. On the other hand, unlike previous works for input saturation like [57, 82-85] that used the sign function with sharp corners at $\left|\tau_{d}\right|=\tau_{M}$, to have all functions being differentiable, the smooth tan-hyperbolic function was employed to bind the input.

To achieve the third goal of our control architecture, careful design of the saturated motor voltage control input, $\boldsymbol{U}(\boldsymbol{u})$, is needed to make the armature current error $\boldsymbol{e}_{3}$, as small as possible. To do this, first let the saturated motor voltage $\boldsymbol{U}$ be $\boldsymbol{U}=\boldsymbol{u}_{\boldsymbol{M}} \times \tanh \left(\boldsymbol{u} / \boldsymbol{u}_{\boldsymbol{M}}\right)$, with $\boldsymbol{u}_{\boldsymbol{M}} \in \mathbf{R}^{\boldsymbol{n}}$ being a known upper bound of $\boldsymbol{u}$, and further let the motor input difference to be $\Delta \boldsymbol{u}=\boldsymbol{U}-\boldsymbol{u}$. Then, choose the following augmented Lyapunov candidate function as,

$$
V_{3}=V_{2}+\frac{1}{2} e_{3}{ }^{T} e_{3}
$$

The time derivative of $V_{3}$ is then given by,

$$
\dot{V}_{3}=\dot{V}_{2}+e_{3}^{T}\left(-R x_{3}-K_{e} x_{2}-d_{a}-L \dot{\tau}_{d r}+u+\Delta u\right)
$$

Design of the intermediate input voltage $\boldsymbol{u}$ can be given by,

$$
\boldsymbol{u}=\boldsymbol{u}_{\boldsymbol{l}}-\boldsymbol{k}_{3} \boldsymbol{e}_{3}-\boldsymbol{K}_{N} \boldsymbol{e}_{2}
$$

where $\boldsymbol{k}_{3}=\operatorname{diag}\left[\boldsymbol{k}_{31}, \ldots, \boldsymbol{k}_{3 n}\right]>0$ are constant parameters, and,

$$
\boldsymbol{u}_{\boldsymbol{l}}=\hat{\boldsymbol{W}}_{2}{ }^{\boldsymbol{T}} \boldsymbol{h}_{2}-\hat{\boldsymbol{D}}_{\boldsymbol{a}} \tanh \left(\frac{\boldsymbol{e}_{3}}{\boldsymbol{\eta}_{3}}\right)
$$

where $\hat{\boldsymbol{W}}_{2}=\left[\hat{\boldsymbol{W}}_{21}, \ldots, \hat{\boldsymbol{W}}_{2 n}\right]^{T} \in \mathbf{R}^{n \times n}$ is the estimation of ideal weight $\boldsymbol{W}_{2}^{*} \in \mathbf{R}^{n \times n}$ of the NNs. Also, defining, $\boldsymbol{R} \boldsymbol{x}_{3}+\boldsymbol{K}_{\boldsymbol{e}} \boldsymbol{x}_{2}+\boldsymbol{L} \dot{\tau}_{d r}-\Delta \boldsymbol{u}=\boldsymbol{W}_{2}^{*} \boldsymbol{h}_{2}\left(\boldsymbol{Z}_{2}\right)+\boldsymbol{\varepsilon}_{2}$, where $\boldsymbol{\varepsilon}_{2}$ is bounded by unknown constant $\overline{\boldsymbol{\varepsilon}}_{2}>0$ as $\left|\boldsymbol{\varepsilon}_{2}\right| \leq \overline{\boldsymbol{\varepsilon}}_{2}$. The input vector $\boldsymbol{Z}_{2} \in \mathbf{R}^{\boldsymbol{n} \times 5}$ is chosen as $\boldsymbol{Z}_{2}=\left[\boldsymbol{x}_{1}{ }^{T}, \boldsymbol{x}_{2}{ }^{T}, \boldsymbol{x}_{3}{ }^{T}, \boldsymbol{\alpha}^{\boldsymbol{T}}, \mathrm{T}_{\boldsymbol{d}}{ }^{\boldsymbol{T}}\right] ; \hat{\boldsymbol{D}}_{\boldsymbol{a}} \in \mathbf{R}^{\boldsymbol{n}}$ are the estimations of the unknown finite number $\boldsymbol{D}_{\boldsymbol{a}} \in \mathbf{R}^{\boldsymbol{n}}$, where $\left|\boldsymbol{\varepsilon}_{2 i}+\overline{\boldsymbol{d}}_{\boldsymbol{a} i}\right|<\boldsymbol{D}_{\boldsymbol{a} i}$ for $\boldsymbol{i}=1, \ldots, \boldsymbol{n} . \boldsymbol{\eta}_{3}>0$ is a small positive number. Other parameters of NNs are the same with the previous section.

To cope with the uncertainties, and unknown parameters in our control design, the Lyapunov function is further modified by choosing an inclusive Lyapunov function candidate as, 


$$
\boldsymbol{V}=\boldsymbol{V}_{3}+\frac{1}{2} \sum_{i=1}^{n} \tilde{\boldsymbol{W}}_{1 i}{ }^{T} \Gamma_{1 i}{ }^{-1} \tilde{\boldsymbol{W}}_{1 i}+\frac{1}{2} \sum_{i=1}^{n} \tilde{\boldsymbol{W}}_{2 i}{ }^{T} \Gamma_{2 i}{ }^{-1} \tilde{\boldsymbol{W}}_{2 i}+\sum_{i=1}^{n} \frac{1}{2} \tilde{\boldsymbol{D}}_{r i}{ }^{T} \tilde{\boldsymbol{D}}_{r i}+\sum_{i=1}^{n} \frac{1}{2} \tilde{\boldsymbol{D}}_{a i}{ }^{T} \tilde{\boldsymbol{D}}_{a i}
$$

where $\tilde{\boldsymbol{W}}_{1 i}=\hat{\boldsymbol{W}}_{1 i}-\boldsymbol{W}_{1 i}{ }^{*}, \tilde{\boldsymbol{W}}_{2 i}=\hat{\boldsymbol{W}}_{2 i}-\boldsymbol{W}_{2 i}{ }^{*}$, and $\left\|\tilde{\boldsymbol{W}}_{1}\right\| \leq \boldsymbol{\varepsilon}_{1},\left\|\tilde{\boldsymbol{W}}_{2}\right\| \leq \boldsymbol{\varepsilon}_{2} ; \tilde{\boldsymbol{D}}_{r i}=\hat{\boldsymbol{D}}_{\boldsymbol{r}}-\boldsymbol{D}_{\boldsymbol{r}}, \tilde{\boldsymbol{D}}_{a i}=\hat{\boldsymbol{D}}_{a i}-\boldsymbol{D}_{a i}$ and $\Gamma_{1 i}=\Gamma_{1 i}{ }^{T}>0, \Gamma_{2 i}=\Gamma_{2 i}{ }^{T}>0$ for $\boldsymbol{i}=1, \ldots, \boldsymbol{n}$. The time derivative of (22) can be written as,

$$
\dot{\boldsymbol{V}}=\dot{\boldsymbol{V}}_{3}+\sum_{i=1}^{n} \tilde{\boldsymbol{W}}_{1 i}^{T} \Gamma_{1 i}{ }^{-1} \dot{\hat{\boldsymbol{W}}}_{1 i}+\sum_{i=1}^{n} \tilde{\boldsymbol{W}}_{2 i}{ }^{T} \Gamma_{2 i}{ }^{-1} \dot{\hat{\boldsymbol{W}}}_{2 i}+\sum_{i=1}^{n} \tilde{\boldsymbol{D}}_{r i} \dot{\hat{\boldsymbol{D}}}_{r i}+\sum_{i=1}^{n} \tilde{\boldsymbol{D}}_{\boldsymbol{a} i} \dot{\hat{\boldsymbol{D}}}_{a i}
$$

The adaptive laws for $\hat{W}_{1 i}, \hat{W}_{2 i}, \hat{D}_{r i}$ and $\hat{D}_{a i}$ are designed as,

$$
\begin{aligned}
& \dot{\hat{\boldsymbol{W}}}_{1 i}=-\Gamma_{1 i}\left(\boldsymbol{e}_{2 i} \boldsymbol{h}_{1 i}+\sigma_{1 i} \hat{\boldsymbol{W}}_{1 i}\right), \\
& \dot{\hat{\boldsymbol{W}}}_{2 i}=-\Gamma_{2 i}\left(\boldsymbol{e}_{3 i} \boldsymbol{h}_{2 i}+\boldsymbol{\sigma}_{2 i} \hat{\boldsymbol{W}}_{2 i}\right), \\
& \dot{\hat{\boldsymbol{D}}}_{\boldsymbol{r}}=\boldsymbol{e}_{2 i} \tanh \left(\frac{\boldsymbol{e}_{2 i}}{\boldsymbol{\eta}_{1}}\right)-\boldsymbol{\sigma}_{r i} \hat{\boldsymbol{D}}_{r i}, \\
& \dot{\hat{\boldsymbol{D}}}_{a i}=\boldsymbol{e}_{3 i} \tanh \left(\frac{\boldsymbol{e}_{3 i}}{\boldsymbol{\eta}_{3}}\right)-\boldsymbol{\sigma}_{a i} \hat{\boldsymbol{D}}_{a i} .
\end{aligned}
$$

The second terms of each adaption law contains the $\sigma$-modification constant which is designed for improving the robustness of the system. Note that without these terms, the estimated parameters would only derive in terms of error functions, which may decrease of the robustness of the system. These terms will also be employed for proving the closed-loop system stability.

Substituting (17) into (16), (21) into (20), (24) into (23), and considering (11), (15), and (19), then (23) can be formed as,

$$
\begin{aligned}
& \dot{\boldsymbol{V}}=-\sum_{i=1}^{n} \boldsymbol{k}_{1 i} \boldsymbol{k}_{b i}^{2} \xi_{i}{ }^{2} \sec ^{2}\left(\frac{\pi}{2} \xi_{i}{ }^{2}\right)-\boldsymbol{e}_{2}{ }^{T} \boldsymbol{k}_{2} \boldsymbol{e}_{2}-\boldsymbol{e}_{3}{ }^{T} \boldsymbol{k}_{3} \boldsymbol{e}_{3} \\
& +\boldsymbol{e}_{2}^{T}\left(\tilde{\boldsymbol{W}}_{1}^{T} \boldsymbol{h}_{1}+\boldsymbol{\varepsilon}_{1}-\boldsymbol{d}_{\boldsymbol{r}}-\left(\boldsymbol{D}_{\boldsymbol{r}}+\tilde{\boldsymbol{D}}_{\boldsymbol{r}}\right) \tanh \left(\frac{\boldsymbol{e}_{2}}{\boldsymbol{\eta}_{1}}\right)-\boldsymbol{f}-\overline{\boldsymbol{f}} \tanh \left(\frac{\boldsymbol{e}_{2}}{\boldsymbol{\eta}_{2}}\right)\right) \\
& +\boldsymbol{e}_{3}{ }^{T}\left(\tilde{\boldsymbol{W}}_{2}{ }^{T} \boldsymbol{h}_{2}+\boldsymbol{\varepsilon}_{2}-\boldsymbol{d}_{\boldsymbol{a}}-\left(\boldsymbol{D}_{\boldsymbol{a}}+\tilde{\boldsymbol{D}}_{\boldsymbol{a}}\right) \tanh \left(\frac{\boldsymbol{e}_{3}}{\boldsymbol{\eta}_{3}}\right)\right)-\sum_{i=1}^{n} \tilde{\boldsymbol{W}}_{1 i}{ }^{T} \boldsymbol{e}_{2 i} \boldsymbol{h}_{1 i}-\sum_{i=1}^{n} \tilde{\boldsymbol{W}}_{2 i}{ }^{T} \boldsymbol{e}_{3 i} \boldsymbol{h}_{2 i}-\sum_{i=1}^{n} \boldsymbol{\sigma}_{1 i} \tilde{\boldsymbol{W}}_{1 i}{ }^{T} \hat{\boldsymbol{W}}_{1 i} \\
& -\sum_{i=1}^{n} \boldsymbol{\sigma}_{2 i} \tilde{\boldsymbol{W}}_{2 i}{ }^{T} \hat{\boldsymbol{W}}_{2 i}+\sum_{i=1}^{n} \tilde{\boldsymbol{D}}_{r i} \boldsymbol{e}_{2 i}{ }^{T} \tanh \left(\frac{\boldsymbol{e}_{2 i}}{\boldsymbol{\eta}_{1}}\right)+\sum_{i=1}^{n} \tilde{\boldsymbol{D}}_{a i} \boldsymbol{e}_{3 i}{ }^{T} \tanh \left(\frac{\boldsymbol{e}_{3 i}}{\boldsymbol{\eta}_{3}}\right)-\sum_{i=1}^{n} \boldsymbol{\sigma}_{r i} \tilde{\boldsymbol{D}}_{r i} \hat{\boldsymbol{D}}_{r i}-\sum_{i=1}^{n} \boldsymbol{\sigma}_{a i} \tilde{\boldsymbol{D}}_{a i} \hat{\boldsymbol{D}}_{a i} .
\end{aligned}
$$

Using Lemma 3 one can obtain, 


$$
\begin{aligned}
& \left|\boldsymbol{e}_{2 i}\right| \boldsymbol{D}_{r i}-\boldsymbol{e}_{2 i} \boldsymbol{D}_{r i} \tanh \left(\frac{\boldsymbol{e}_{2 i}}{\eta_{1}}\right) \leq \boldsymbol{k}_{p} \boldsymbol{D}_{r i} \boldsymbol{\eta}_{1}, \\
& \left|\boldsymbol{e}_{3 i}\right| \boldsymbol{D}_{a i}-\boldsymbol{e}_{3 i} \boldsymbol{D}_{a i} \tanh \left(\frac{\boldsymbol{e}_{3 i}}{\eta_{3}}\right) \leq \boldsymbol{k}_{\boldsymbol{p}} \boldsymbol{D}_{a i} \boldsymbol{\eta}_{3},
\end{aligned}
$$

and

$$
\left|\boldsymbol{e}_{2 i}\right| \overline{\boldsymbol{f}}-\boldsymbol{e}_{2 i} \overline{\boldsymbol{f}} \tanh \left(\frac{\boldsymbol{e}_{2 i}}{\eta_{2}}\right) \leq \boldsymbol{k}_{\boldsymbol{p}} \overline{\boldsymbol{f}} \boldsymbol{\eta}_{2}
$$

In addition, the following inequality can be given by completion of squares,

$$
-\sigma_{i} \tilde{W}_{i}^{T} \hat{W}_{i} \leq-\frac{\sigma_{i}}{2}\left\|\tilde{W}_{i}\right\|^{2}+\frac{\sigma_{i}}{2}\left\|W_{i}^{*}\right\|^{2}
$$

and

$$
-\frac{\sigma_{i}}{2}\left\|\tilde{W}_{i}\right\|^{2} \leq-\frac{1}{2} \sum_{i=1}^{3} \frac{\sigma_{i}}{\lambda_{\max }\left(\Gamma_{i}^{-1}\right)} \tilde{W}_{i}^{T} \Gamma_{i}^{-1} \tilde{W}_{i} .
$$

It can also be written,

$$
-\sigma_{i} \tilde{D}_{i} \hat{D}_{i}=-\sigma_{i} \tilde{D}_{i}\left(\tilde{D}_{i}+D_{i}\right) \leq-\frac{\sigma_{i}}{2} \tilde{D}_{i}^{2}+\frac{\sigma_{i}}{2} D_{i}^{2}
$$

Finally, using (25), and applying Lemma 1, and employing (26) - (30), it can be shown that,

$$
\begin{aligned}
& \dot{\boldsymbol{V}} \leq-\sum_{i=1}^{n} \boldsymbol{k}_{1 i} \boldsymbol{k}_{b i}^{2} \xi_{i}^{2} \Lambda_{i} \sec ^{2}\left(\frac{\pi}{2} \xi_{i}^{2}\right)-\boldsymbol{e}_{2}{ }^{T} \boldsymbol{k}_{2} \boldsymbol{e}_{2}-\boldsymbol{e}_{3}{ }^{T} \boldsymbol{k}_{3} \boldsymbol{e}_{3}-\frac{1}{2} \sum_{i=1}^{n} \frac{\sigma_{1 i}}{\lambda_{\max }\left(\Gamma_{1 i}{ }^{-1}\right)} \tilde{\boldsymbol{W}}_{1 i}{ }^{T} \Gamma_{1 i}{ }^{-1} \tilde{\boldsymbol{W}}_{1 i} \\
& -\frac{1}{2} \sum_{i=1}^{n} \frac{\sigma_{2 i}}{\lambda_{\max }\left(\Gamma_{2 i}{ }^{-1}\right)} \tilde{\boldsymbol{W}}_{2 i}{ }^{T} \Gamma_{2 i}{ }^{-1} \tilde{\boldsymbol{W}}_{2 i}-\frac{1}{2} \sum_{i=1}^{n} \boldsymbol{\sigma}_{r i} \tilde{\boldsymbol{D}}_{r i}{ }^{2}-\frac{1}{2} \sum_{i=1}^{n} \boldsymbol{\sigma}_{a i} \tilde{\boldsymbol{D}}_{a i}{ }^{2}+\frac{1}{2} \sum_{i=1}^{n} \sigma_{1 i}\left\|\boldsymbol{W}^{*}{ }_{1 i}\right\|^{2} \\
& +\frac{1}{2} \sum_{i=1}^{n} \sigma_{2 i}\left\|W_{2 i}^{*}\right\|^{2}+\frac{1}{2} \sum_{i=1}^{n} \sigma_{r i} D_{r i}{ }^{2}+\frac{1}{2} \sum_{i=1}^{n} \sigma_{a i} D_{a i}{ }^{2}+\sum_{i=1}^{n} k_{p} \eta_{1} D_{r i}+\sum_{i=1}^{n} k_{p} \eta_{3} D_{a i}+k_{p} \bar{f} \eta_{2} \\
& \leq-\boldsymbol{v}_{1} \boldsymbol{V}+\boldsymbol{v}_{2} \text {, }
\end{aligned}
$$

where, $v_{1}$, and $v_{2}$ are defined as,

$$
\begin{aligned}
& \boldsymbol{v}_{1}=\min \left(\boldsymbol{k}_{1 i}, 2 \frac{\lambda_{\min }\left(\boldsymbol{k}_{2}\right)}{\lambda_{\max }(\boldsymbol{M})}, 2 \frac{\lambda_{\min }\left(\boldsymbol{k}_{3}\right)}{\lambda_{\max }(\boldsymbol{L})}, \frac{\sigma_{1 i}}{\lambda_{\max }\left(\Gamma_{1 i}{ }^{-1}\right)}, \frac{\boldsymbol{\sigma}_{2 i}}{\lambda_{\max }\left(\Gamma_{2 i}{ }^{-1}\right)}, \boldsymbol{\sigma}_{r i}, \boldsymbol{\sigma}_{a i}\right), \\
& \boldsymbol{v}_{2}=1 / 2\left(\sum_{i=1}^{n} \sigma_{1 i}\left\|W_{1 i}^{*}\right\|^{2}+\sum_{i=1}^{n} \sigma_{2 i}\left\|W_{2 i}^{*}\right\|^{2}+\sum_{i=1}^{n} \sigma_{r i} D_{r i}{ }^{2}+\sum_{i=1}^{n} \sigma_{a i} D_{a i}{ }^{2}\right)+\sum_{i=1}^{n} \boldsymbol{k}_{\boldsymbol{p}} \boldsymbol{\eta}_{1} \boldsymbol{D}_{\boldsymbol{r} i}+\sum_{i=1}^{n} \boldsymbol{k}_{\boldsymbol{p}} \boldsymbol{\eta}_{3} \boldsymbol{D}_{\boldsymbol{a} i}+\boldsymbol{k}_{\boldsymbol{p}} \overline{\boldsymbol{f}} \boldsymbol{\eta}_{2}
\end{aligned}
$$


for $\boldsymbol{i}=1, \ldots, n$.

The schematic of the proposed control is depicted in Fig.1.

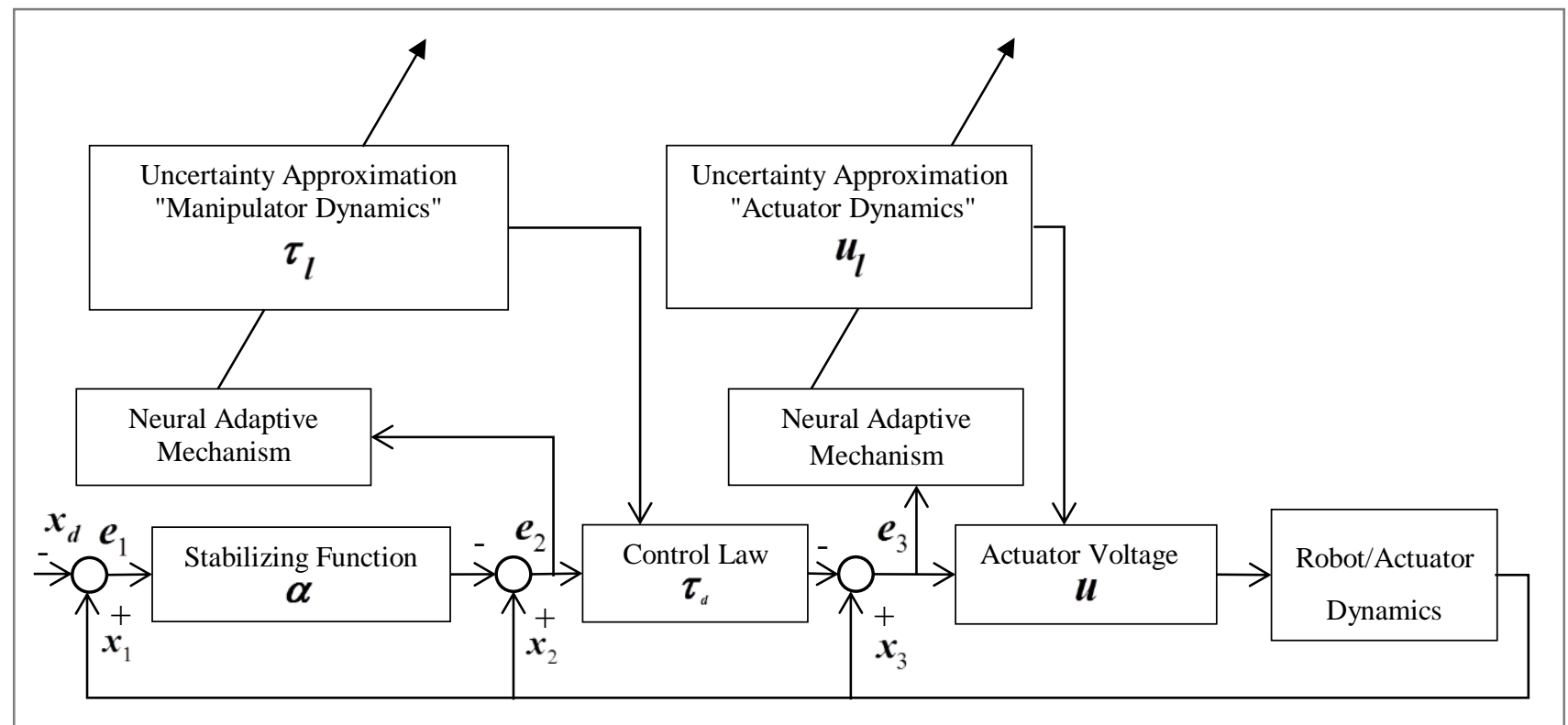

Figure 1. Adaptive NNs control diagram for a robotic system with time-varying constraints.

Theorem 1. For the integrated manipulator dynamics (4), under Assumptions 1- 3, with the proposed control (10), (16), (17), (20), and (21) together with update laws (24), and bounded NNs basis function $\boldsymbol{h}(\boldsymbol{Z})$, and given any initial set defined by,

$$
\Omega_{i}=\left\{\boldsymbol{q}_{1 i}, \boldsymbol{i}=1, \ldots, \boldsymbol{n} \mid \underline{\boldsymbol{k}}_{o i}(0)<\boldsymbol{q}_{1 i}(0)<\overline{\boldsymbol{k}}_{o i}(0)\right\}
$$

and providing that $\hat{\boldsymbol{W}}_{1}(0), \hat{\boldsymbol{W}}_{2}(0), \hat{\boldsymbol{D}}_{\boldsymbol{r}}(0)$, and $\hat{\boldsymbol{D}}_{\boldsymbol{a}}(0)$ are bounded, then the following properties hold:

(i) the error signals $\boldsymbol{e}_{1}, \boldsymbol{e}_{2}$, and $\boldsymbol{e}_{3}$ in the closed-loop system will remain in the compact set defined by,

$$
\Omega_{e}:=\left\{\boldsymbol{e}_{1 i}, \boldsymbol{e}_{2 i}, \boldsymbol{e}_{3 i}, \boldsymbol{i}=1, \ldots, \boldsymbol{n} \mid-\underline{\Delta}_{i} \leq \boldsymbol{e}_{1 i} \leq \bar{\Delta}_{i},\left\|\boldsymbol{e}_{2}\right\| \leq \sqrt{\frac{2 \Phi}{\lambda_{\text {min }}(M)}},\left\|\boldsymbol{e}_{3}\right\| \leq \sqrt{\frac{2 \Phi}{\lambda_{\text {min }}(L)}},\right\},
$$

where $\Phi=\boldsymbol{V}(0)+\boldsymbol{v}_{2} / \boldsymbol{v}_{1}$ and $\bar{\Delta}_{i}=\boldsymbol{k}_{m i} \sqrt{2 \tan ^{-1}\left(\boldsymbol{\pi} \Phi / \boldsymbol{k}_{m i}^{2}\right) / \boldsymbol{\pi}}$ and $\underline{\Delta}_{i}=\boldsymbol{k}_{\boldsymbol{n} i} \sqrt{2 \tan ^{-1}\left(\boldsymbol{\pi} \Phi / \boldsymbol{k}_{n i}^{2}\right) / \boldsymbol{\pi}}$.

(ii) the error signals $\boldsymbol{e}_{1}, \boldsymbol{e}_{2}$, and $\boldsymbol{e}_{3}$ will eventually converge to the compact set defined by,

$$
\Omega_{e U}:=\left\{\boldsymbol{e}_{1 i}, \boldsymbol{e}_{2 i}, \boldsymbol{e}_{3 i}, \boldsymbol{i}=1, \ldots, \boldsymbol{n} \mid-\underline{\Delta}_{i}^{*} \leq \boldsymbol{e}_{1 i} \leq \bar{\Delta}_{i}^{*},\left\|\boldsymbol{e}_{2}\right\| \leq \sqrt{\frac{2 \boldsymbol{v}_{2}}{\boldsymbol{v}_{1} \lambda_{\text {min }}(M)}},\left\|\boldsymbol{e}_{3}\right\| \leq \sqrt{\frac{2 \boldsymbol{v}_{2}}{v_{1} \lambda_{\text {min }}(L)}}\right\},
$$

where $\bar{\Delta}_{i}^{*}=\boldsymbol{k}_{m i} \sqrt{2 \tan ^{-1}\left(\pi \boldsymbol{v}_{2} / \boldsymbol{k}_{m i}^{2} \boldsymbol{\nu}_{1}\right) / \boldsymbol{\pi}}$ and $\underline{\Delta}_{i}^{*}=\boldsymbol{k}_{n i} \sqrt{2 \tan ^{-1}\left(\pi \boldsymbol{v}_{2} / \boldsymbol{k}_{\boldsymbol{n}}^{2} \boldsymbol{\nu}_{1}\right) / \boldsymbol{\pi}}$. 
(iii) The joint space vector $\boldsymbol{q}_{1}$ remains in the constraint set

$$
\Omega_{y}=\left\{\boldsymbol{q}_{i} \in \mathbf{R}, \boldsymbol{i}=1, \ldots, \boldsymbol{n} \mid \underline{\boldsymbol{k}}_{o i}<-\underline{\Delta}_{i}-\underline{\boldsymbol{k}}_{d i} \leq \boldsymbol{q}_{i} \leq \bar{\Delta}_{i}+\overline{\boldsymbol{k}}_{d i}<\overline{\boldsymbol{k}}_{o i}\right\},
$$

i.e. the multiple asymmetric time-varying joint space constraint is never violated.

(iv) All signals of the closed-loop system are bounded.

Proof. Please see Appendix A.

Remark 6. Following the same procedure with $(i)$, it is easy to show that $\boldsymbol{W}_{1}, \boldsymbol{W}_{2}, \boldsymbol{D}_{\boldsymbol{r}}$ and $\boldsymbol{D}_{\boldsymbol{a}}$ are bounded. Accordingly, this development guarantees the stability as being semiglobally uniformly ultimately bounded [81]. From (ii) and following the same line of argument with (iii), the steady state compact set for the joint space vector $\boldsymbol{q}_{1}$ can be written as $\Omega_{f}=\left\{\boldsymbol{q}_{i} \in \mathbf{R}, \boldsymbol{i}=1, \ldots, \boldsymbol{n} \mid-\underline{\Delta}_{i}^{*}-\underline{\boldsymbol{k}}_{d i} \leq \boldsymbol{q}_{i} \leq \bar{\Delta}_{i}^{*}+\overline{\boldsymbol{k}}_{d i}\right\}$. It is obvious that the size of the initial compact set $\Omega_{i}$ affects the bounding compact set $\Omega_{y}$, but not $\Omega_{f}$.

Remark 7. It is clear that by changing the design parameters, the smaller steady state set, $\Omega_{e U}$, can be obtained. This can be achieved by adjusting control parameters to obtain smaller $\boldsymbol{v}_{1}$, and larger $\boldsymbol{v}_{2}$. Namely, i) increasing control matrix $\boldsymbol{k}_{2}, \boldsymbol{k}_{3}$, and control gains $\sigma_{1}, \boldsymbol{\sigma}_{2}, \boldsymbol{\sigma}_{\boldsymbol{r}}, \boldsymbol{\sigma}_{\boldsymbol{a}}$ might help to increase the constant $\boldsymbol{v}_{1}$, and ii) decreasing control gains $\eta_{1}, \eta_{2}, \eta_{3}$, and $\sigma_{1}, \sigma_{2}, \sigma_{r}, \sigma_{a}$ might lead to reducing the constant $\boldsymbol{v}_{2}$. However, as parameters $\hat{\boldsymbol{W}}_{1}$, and $\hat{\boldsymbol{W}}_{2}$ will only be estimated using tracking errors, if $\sigma_{1}$, and $\sigma_{2}$ are chosen to be too small, then using small $\sigma_{1}, \sigma_{2}$ may produce large NNs estimation weights, and similarly small $\sigma_{r}, \sigma_{a}$, may result in to large adapting disturbance parameters, and thus decrease the external disturbance robustness. On the other hand, choosing large $\boldsymbol{k}_{2}, \boldsymbol{k}_{3}$ may lead to the increase in motor input voltage and excite unmodeled dynamics. Accordingly, proper design parameters must be chosen by considering the balance between tracking performance and system stability.

Remark 8. Compared with the previous works on constrained control of manipulator systems using BLFs ([40], and [41]), in the proposed control scheme in this paper, the linearly-in-parameter conditions of the system dynamics are eliminated and unknown actuator dynamics are further incorporated to increase the efficiency. Also, with respect to [45], [23] and [86], in this work the set of feasible initial positions is maximized by incorporating both time-varying and asymmetric barrier limits. Furthermore, different from [70-73], in the presented study, the constraints are dealt with directly and it removes the extra steps on mapping [70], error transformation [11,71], or transforming the constrained system into an unconstrained one $[72,73]$.

\section{Illustrative examples}

In this section, to illustrate that the developed method is effective, numerical simulations are utilized. A 3D revolute-revolute-prismatic robotic manipulator, (see Fig. 2) is selected as an example. The section includes two case studies. The first case study illustrates the tracking performance of the proposed 
control without violating constraints while relaxing different initial conditions. The second case study highlights the ability of the presented method to cope with time varying constrained sets. The detailed system parameters of the studied robotic manipulator model actuated by DC servomotors is chosen as $\boldsymbol{m}_{1}=2 \mathrm{~kg}, \boldsymbol{m}_{2}=1 \mathrm{~kg}, \boldsymbol{m}_{3}=0.2 \mathrm{~kg}, \boldsymbol{L}_{1}=0.35 \mathrm{~m}, \boldsymbol{L}_{2}=0.32 \mathrm{~m}, \boldsymbol{R}=1.6 \boldsymbol{I}_{\boldsymbol{d}} \Omega, \boldsymbol{L}=0.0048 \boldsymbol{I}_{\boldsymbol{d}} \Omega-\boldsymbol{s}$, $\boldsymbol{K}_{e}=0.19 \boldsymbol{I}_{d} \mathrm{~V} / \mathrm{rad} / \mathrm{s}$, and $\boldsymbol{K}_{N}=30 \boldsymbol{I}_{d} \mathrm{Nm} / \boldsymbol{A}$, where $\boldsymbol{I}_{d}$ is the $3 \times 3$ Identity matrix.

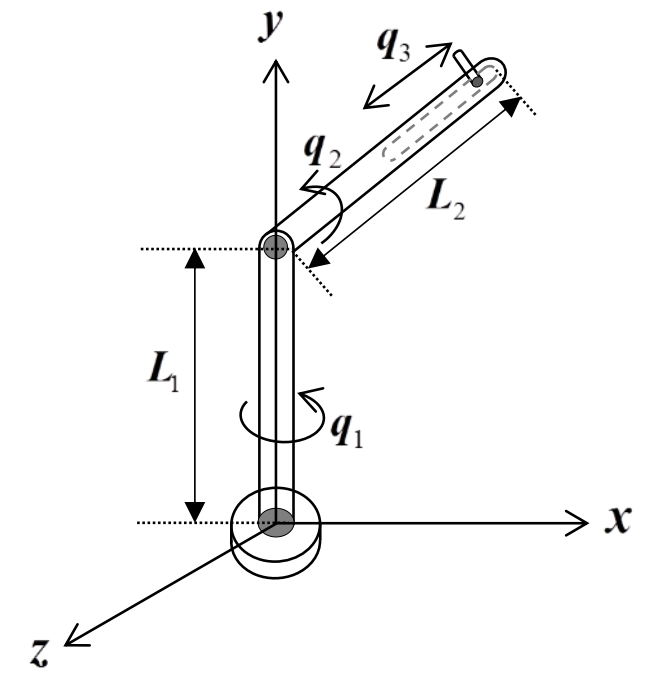

Figure 2. Schematic of the revolute-revolute-prismatic robotic system

The objective of control is so the joints of the robot manipulator $\boldsymbol{q}_{1}(t), \boldsymbol{q}_{2}(t)$, and $\boldsymbol{q}_{3}(\boldsymbol{t})$ track the desired trajectories as $\boldsymbol{x}_{\boldsymbol{d}}=\left[\boldsymbol{q}_{1 d}, \boldsymbol{q}_{2 d}, \boldsymbol{q}_{3 d}\right]^{T}=[\sin (2 \boldsymbol{t}) \exp (-0.2 \boldsymbol{t}), 0.5 \sin (\boldsymbol{t}), 0.2 \sin (\boldsymbol{t})]^{T}$ with $\boldsymbol{t} \in[0,10]$ second without violating the constraints defined as $\underline{\boldsymbol{k}}_{o i}<\boldsymbol{q}_{1 i}<\overline{\boldsymbol{k}}_{o i}, \boldsymbol{i}=1,2,3$. The initial adapting parameters and initial NNs weight estimates are chosen as $\hat{\boldsymbol{D}}_{\boldsymbol{r}}(0)=\hat{\boldsymbol{D}}_{\boldsymbol{a} i}(0)=\hat{\boldsymbol{W}}_{1 i}(0)=\hat{\boldsymbol{W}}_{2 i}(0)=0.1$, for $\boldsymbol{i}=1,2$ and 3. In addition, for bounding $\tau_{d}$, and $\boldsymbol{u}$, we consider $\tau_{M}=\boldsymbol{u}_{M}=[30,30,20]^{T}$. The external disturbances are considered as $\quad \boldsymbol{d}_{r}=[2 \sin (\boldsymbol{t}), 3\|\boldsymbol{q}\|, 6\|\boldsymbol{\boldsymbol { q }}\|]^{T} \quad$ and $\boldsymbol{d}_{\boldsymbol{a}}=[\exp (-2 \boldsymbol{t}), 0.2 \sin (\boldsymbol{t}), 0.5 \exp (-5 t)]^{T}$, and the interaction force vector is defined as $\boldsymbol{f}=\left[2 \sin \left(\boldsymbol{q}_{1}\right), 2 \cos \left(\boldsymbol{q}_{2}\right), \sin \left(\boldsymbol{q}_{3}\right)\right]^{T}$ which is bounded by $\overline{\boldsymbol{f}}=[2,2,1]^{T}$. For the simulation, the control gains are selected as $\boldsymbol{k}_{1}=3 \boldsymbol{I}_{\boldsymbol{d}}, \boldsymbol{k}_{2}=\boldsymbol{k}_{3}=\boldsymbol{I}_{\boldsymbol{d}}$. Other control parameters are chosen as $\Gamma_{1 i}=\Gamma_{2 i}=100$, $\sigma_{1 i}=\sigma_{2 i}=\sigma_{r i}=\sigma_{a i}=0.05$, and $\eta_{i}=0.1$ for $\boldsymbol{i}=1,2$, and 3. Also, the NNs with ten nodes on each hidden layer with the center $\vartheta_{i}$ uniformly distributed in [-3,3], with the width being $\psi_{i}=10$ are selected. The joint space constraints are written in the form,

$$
\begin{aligned}
& \overline{\boldsymbol{k}}_{o i}=\boldsymbol{a}_{u i} \exp (-\boldsymbol{t})+\boldsymbol{q}_{d i}+\boldsymbol{a}_{o i}, \\
& \underline{\boldsymbol{k}}_{o i}=-\boldsymbol{a}_{l i} \exp (-\boldsymbol{t})+\boldsymbol{q}_{d i}-\boldsymbol{a}_{o i},
\end{aligned}
$$

for $\boldsymbol{i}=1,2, \ldots, 3$, where $\boldsymbol{q}_{\boldsymbol{d i}}$, and $\boldsymbol{a}_{\boldsymbol{o} i}$ denote the desired trajectory, and the required constraint values of 
the $i^{\text {th }}$ joint, respectively; $\boldsymbol{a}_{u i}$, and $\boldsymbol{a}_{l i}$ can be defined according to the initial conditions. Accordingly, using the above asymmetric time-varying constraints, the constraint boundaries can cover any initial conditions, i.e. all $\boldsymbol{q}_{1 i} \in \Omega_{i}$, and they then exponentially tend to be close to the desired trajectories as $\lim _{\boldsymbol{t} \rightarrow 0} \boldsymbol{a}_{\boldsymbol{u}} \exp (-\boldsymbol{t})=\boldsymbol{a}_{\boldsymbol{u}}$, and $\lim _{\boldsymbol{t} \rightarrow 0} \boldsymbol{a}_{l i} \exp (-\boldsymbol{t})=\boldsymbol{a}_{\boldsymbol{l}}$, and $\lim _{\boldsymbol{t} \rightarrow \infty} \boldsymbol{a}_{\boldsymbol{u}} \exp (-\boldsymbol{t})=\lim _{\boldsymbol{t} \rightarrow \infty} \boldsymbol{a}_{l i} \exp (-\boldsymbol{t})=0$.

Remark 9. Developing the asymmetric time-varying constraint can relax any initial condition and tend to the specific distance with the desired trajectory for the rest of the movement, while the constraints presented in most of previous works like [45, 87-89] are assumed to remain symmetric and constant which is not an advantageous assumption in practice. Note that using symmetric and timeinvariant constraints may also have some inefficiency for the initial condition which is far from the desired trajectory. In that case, the designer has to choose a constant constraint which is far from the desired trajectory and keep it constant with the rest of the movement. Accordingly, due to the probable large distance from the constraint with the real trajectory, such a constraint may be useless in practice specifically for states which are not located on the matching side with the desired trajectory.

Remark 10. In several real applications, we can define the desired trajectory according to the design characteristics, and then by choosing the proper values of the desired distance, $\boldsymbol{a}_{\boldsymbol{o}}$, the preferred constrained control can be satisfied. For example, in the upper-limb robotic rehabilitation, $\boldsymbol{q}_{\boldsymbol{d}}$ can be defined according to the physical characteristics of the patient, and then by choosing proper amounts of $\boldsymbol{a}_{\boldsymbol{o}}$, the safe tracking control can be achieved.

\subsection{First case study}

In this case study, we show the ability of the proposed method to tackle asymmetric time-varying constraints within different initial conditions. It is demonstrated that by defining constraint regions as in (33), and using the proposed method, that the error variables converged to small neighborhoods of zero, and the constrained sets are not transgressed, provided that the initial states are feasible. The initial conditions are selected as $\boldsymbol{q}(0)=[-1.8,0.8,-0.6]^{T}, \quad \dot{\boldsymbol{q}}(0)=[0,0,0]^{T}$ and $\boldsymbol{I}(0)=[0.1,0.1,0.1]^{T}$. Moreover, the following constraint parameters are chosen, $\boldsymbol{a}_{1 \boldsymbol{u}}=0, \boldsymbol{a}_{2 \boldsymbol{u}}=0.8, \boldsymbol{a}_{3 \boldsymbol{u}}=0$, and $\boldsymbol{a}_{1 l}=1.8, \boldsymbol{a}_{2 l}=0, \boldsymbol{a}_{3 l}=0.6$ with $\boldsymbol{a}_{\boldsymbol{o} 1}=\boldsymbol{a}_{\boldsymbol{o} 2}=\boldsymbol{a}_{\boldsymbol{o} 3}=0.2$. Note that the magnitudes of $\boldsymbol{a}_{\boldsymbol{u} i}$, and $\boldsymbol{a}_{l i}$ are taken from the initial conditions. The simulation figures are listed in Figs. 3-6.

The tracking performance of the controller is shown in Fig.3. The figure shows that the proposed controller effectively tracks the given desired trajectories and the controller does not violate the set of time-varying constraints. As shown in this figure, using (33), the constraints are set so that they can be enlarged enough to cover the initial conditions. Thus, the controller is able to handle any initial conditions within the constrained regions by selecting proper constraint parameters. Figure 4 shows the control inputs. It is clear that the joint torques and the motor input voltages are saturated, while the control performance is satisfactory. The system errors converge to close to zero, as in Fig. 5. It can be seen from the figures that all errors converge to near zero within 2 seconds. Also, as it is shown in Fig. 5, due to imposing constraints on positions, the maximum values of the position errors are bound using the proposed method. The radial basis function NNs weights in the sense of two-norm are shown in Fig. 6. As shown in this figure these parameters are all bounded. 


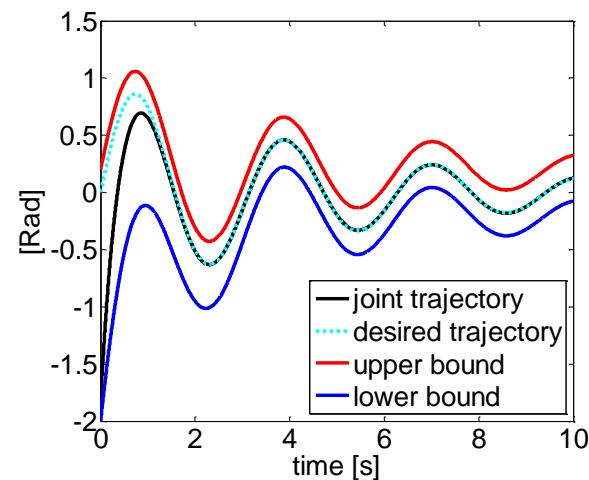

a. first joint

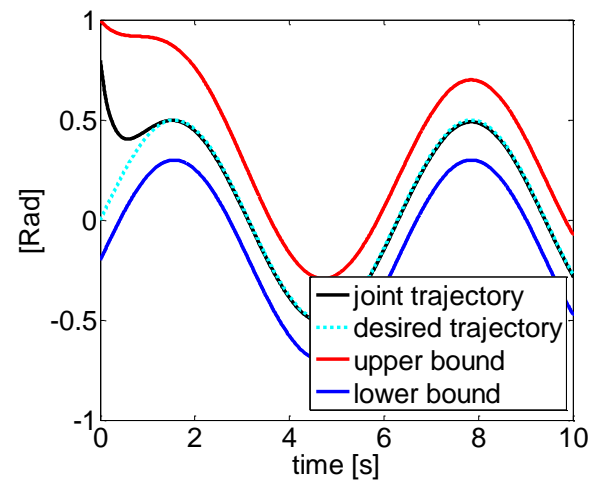

b. second joint

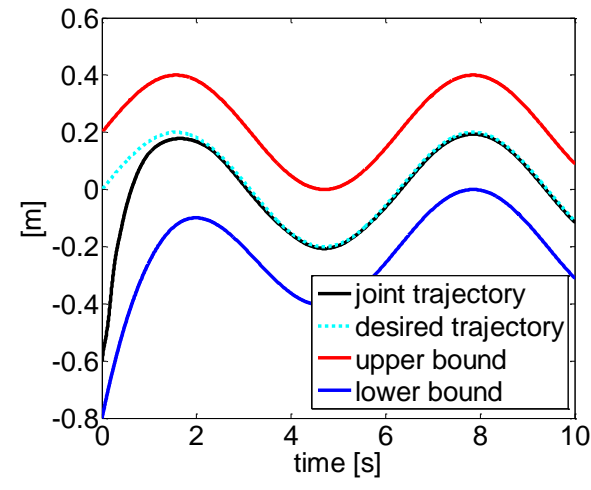

c. third joint

Figure 3. Position of joints with upper and lower bounds

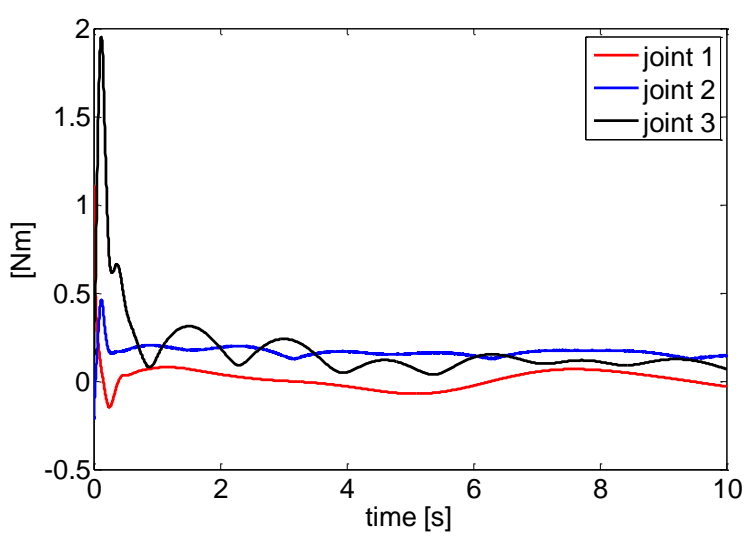

a. joint control torques

Figure 4. Control inputs
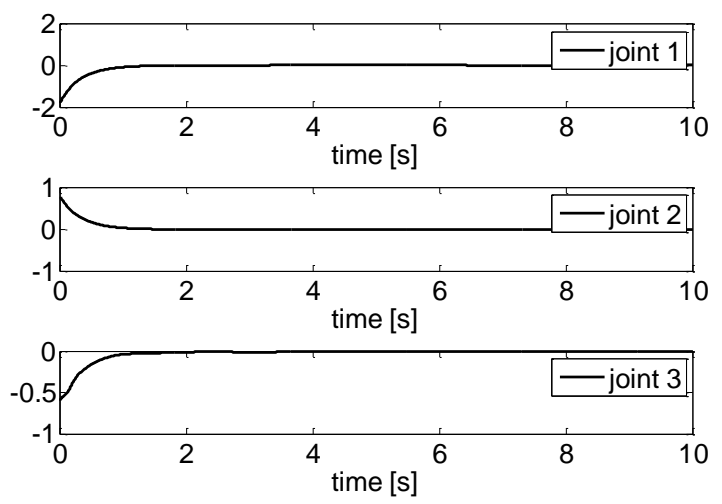

a. errors in the position tracking

Figure 5. Tracking errors

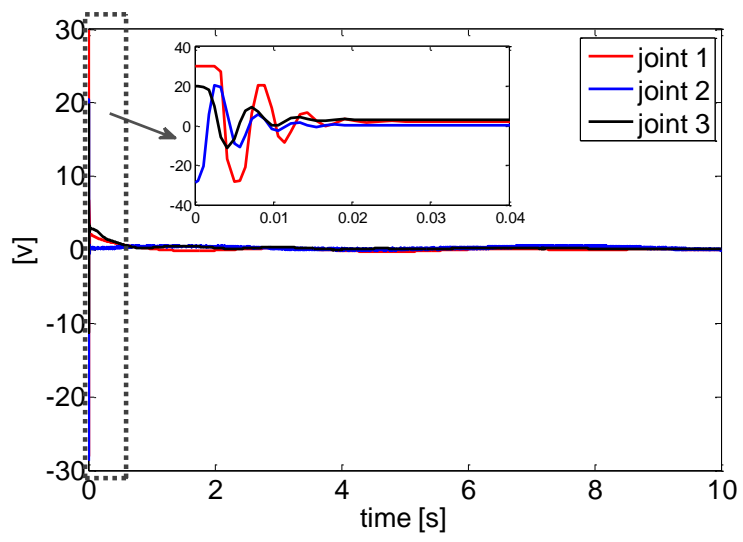

b. input voltages
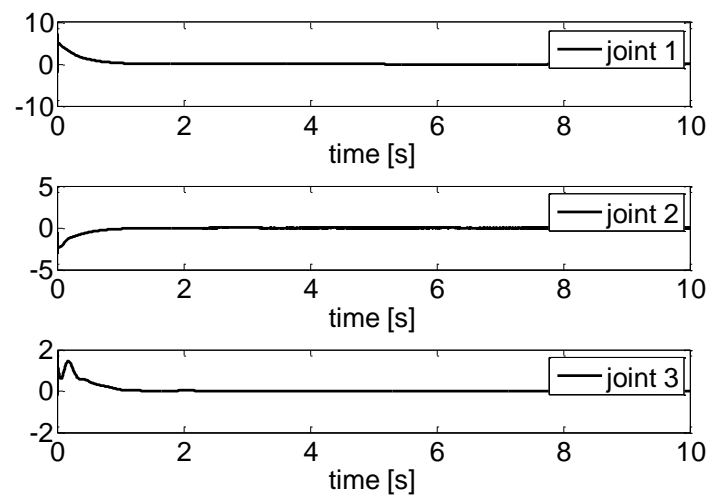

b. errors in the velocity tracking 

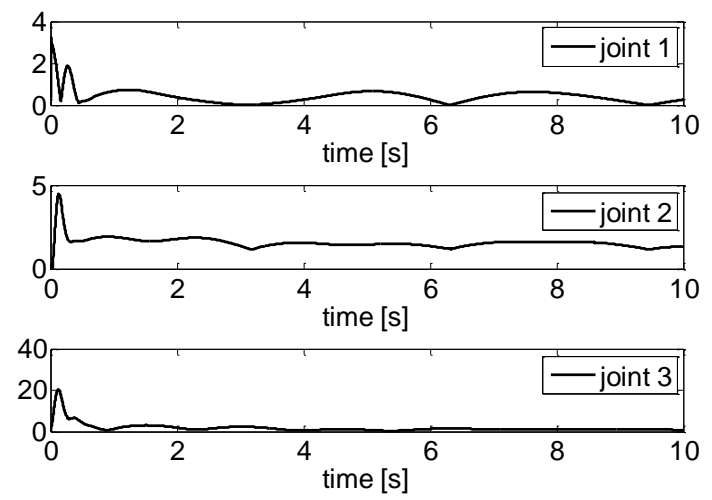

a. first NNs weights $\left(\left\|\hat{W}_{1}\right\|\right)$
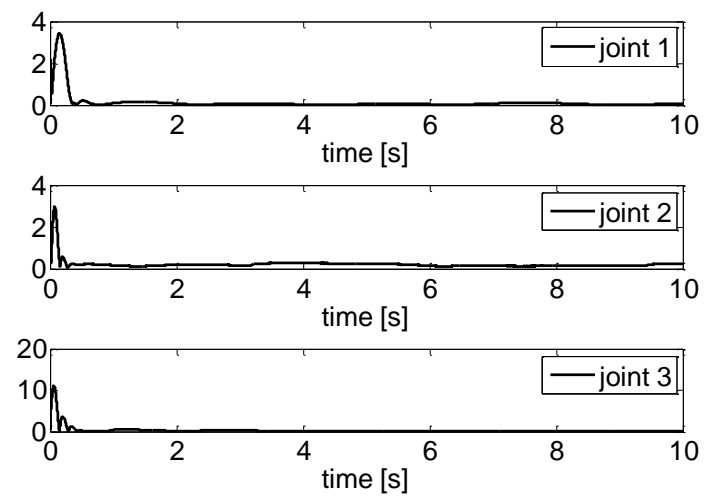

b. second NNs weights $\left(\left\|\hat{\boldsymbol{W}}_{2}\right\|\right)$

Figure 6. Norms of radial basis functions NNs weights

\subsection{Second case study}

In this case study, the simulation is performed to highlight the effectiveness of the proposed method to provide a constrained behavior where variables are growing close to their bounds. To this end, we render smaller ranges for constrained error sets. Accordingly, the magnitude of constraint parameters $\boldsymbol{a}_{0 i}$ are decreased to $\boldsymbol{a}_{\boldsymbol{0} 1}=0.005, \boldsymbol{a}_{\boldsymbol{\prime} 2}=0.01$, and $\boldsymbol{a}_{\boldsymbol{o} 3}=0.008$. Also, the initial conditions for the position are chosen as $\boldsymbol{q}(0)=[-0.1,0.1,-0.1]^{T}$ to be close to the desired trajectory. Accordingly, the corresponding constraint parameters on (33) are selected as $\boldsymbol{a}_{1 u}=\boldsymbol{a}_{2 l}=\boldsymbol{a}_{3 u}=0$, and $\boldsymbol{a}_{1 l}=\boldsymbol{a}_{2 u}=\boldsymbol{a}_{3 l}=0.1$. The execution time is increased to $t=25$ seconds, as well. Other simulation parameters are the same for the first simulation. The simulation figures are illustrated in Figs. 7-9.

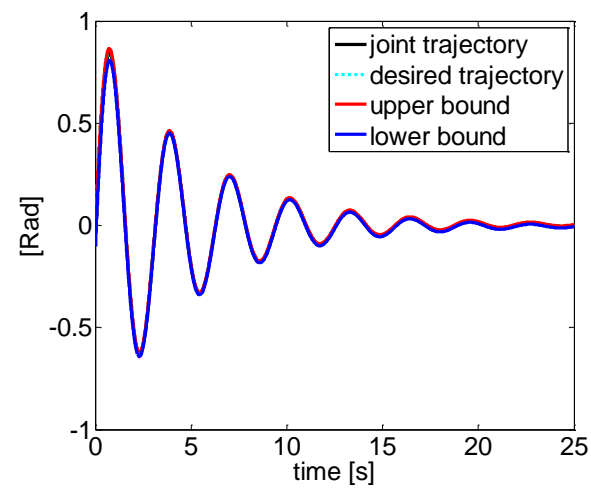

a. first joint

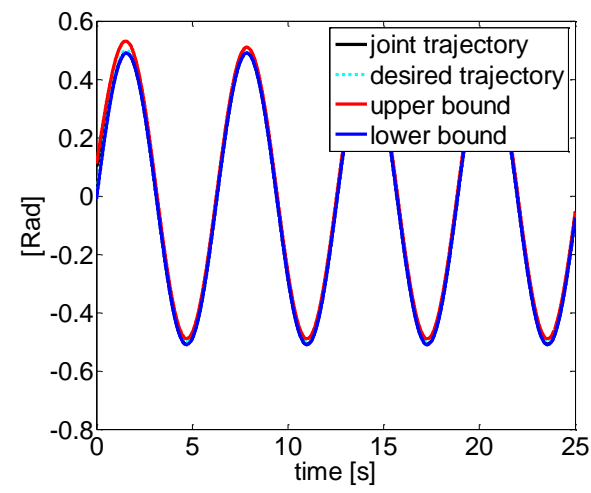

b. second joint

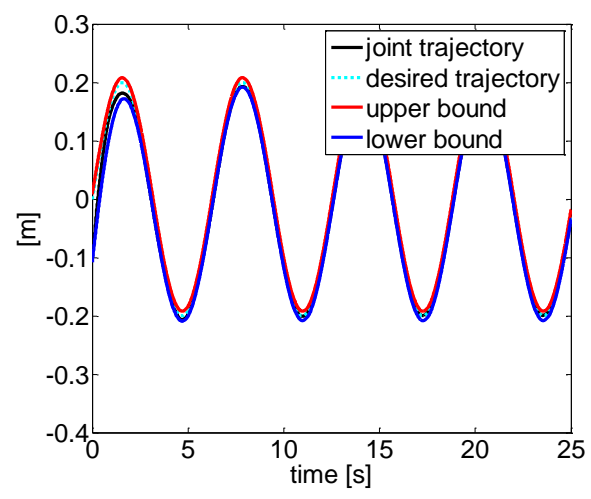

c. third joint

Figure 7. Constrained tracking of positions 


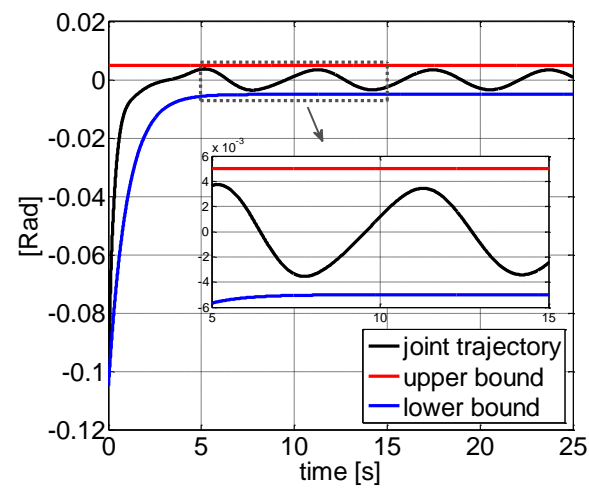

a. first joint

Figure 8. Position tracking errors

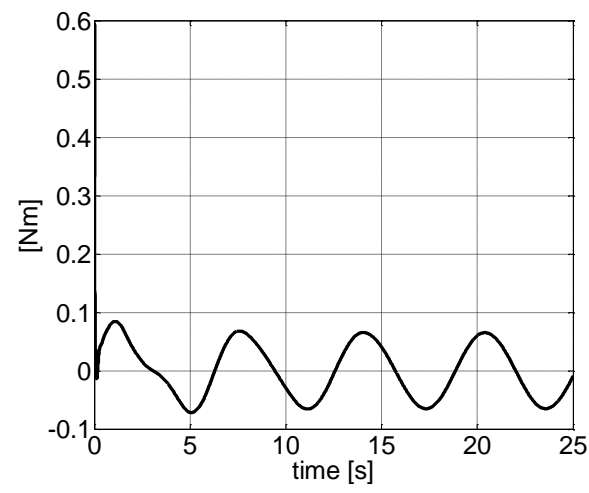

a. first joint

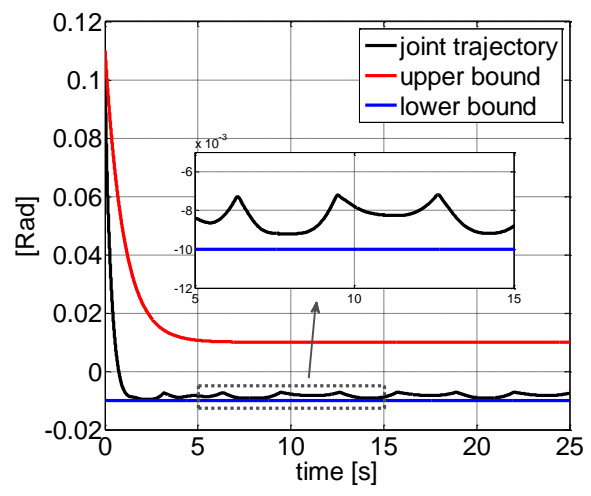

b. second joint

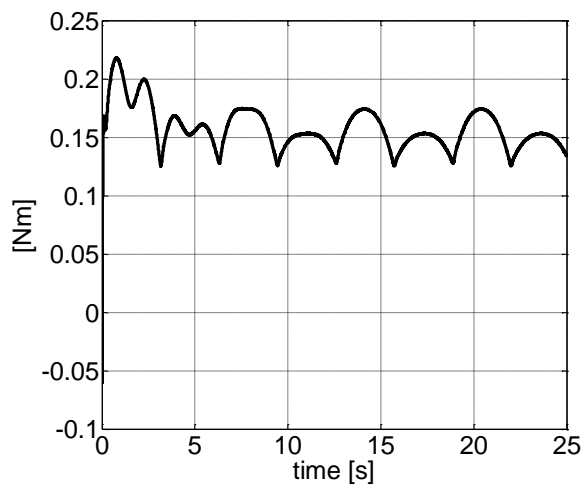

b. second joint

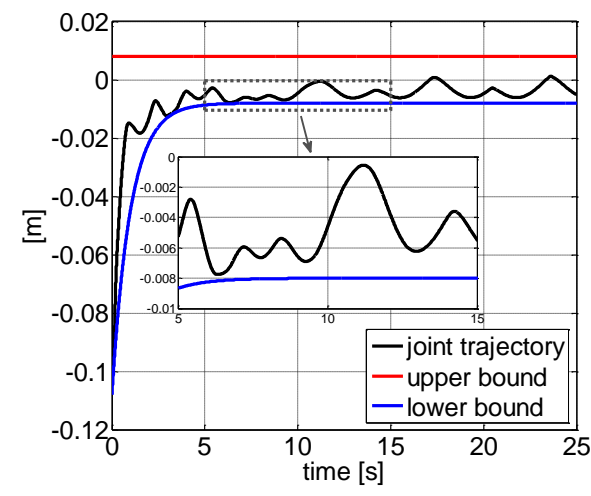

c. third joint

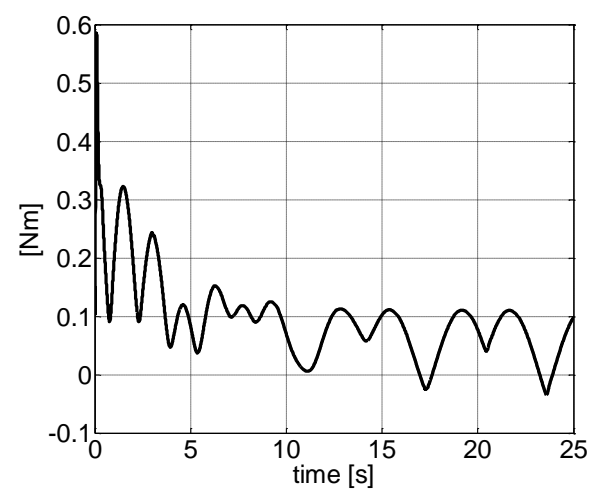

c. third joint

Figure 9. Joint control signals

The tracking performance of the constrained robotic controller is illustrated in Figs 7, 8. It is observed from Fig. 7 that all signals track the desired trajectories successfully. Figure 8, shows position tracking errors. As it is obvious in this figure, error signals never violate the constraints even if errors are growing close to their bounds. The control input signal $\tau_{d}$ is illustrated in Fig. 9. It can be observed by considering Fig 8, and Fig. 9 together that the control inputs grow to their peak values as the tracking error approaches their constraint boundaries. Thus, it provides larger control signals to prevent violation of the constraints. The tangent tvBLFs as discussed in the Introduction and the control design is responsible for such control effects. It can be observed from Figs. 7-9 that under the proposed tangent tvBLFs controller good tracking performance is achieved while the error signals never transgress the constraint sets.

\section{Conclusion}

In this paper, a neural adaptive barrier control was developed for an uncertain robot subject to timevarying joint space constraints. External disturbances, unknown interaction force, saturation of input signals, and uncertainties in both structural dynamics and actuator dynamics were considered, and the asymmetric tangent tvBLFs were employed to prevent the constraint violation. Appropriate NN weight update laws were designed to compensate for the uncertainties and to improve the system robustness. It was proven that multiple asymmetric time-varying joint constraints would not be violated and that the signals of the closed-loop system were bounded. The theoretical analysis has verified the performance of 
the proposed control in tracking the desired trajectory subject to time-varying joint constraints. Then, the effectiveness of the theoretical results was illustrated by performing numerical simulations. Future research directions may include an extension of the constraints on the joint rate variables. Also, the presented control can be integrated with the impedance controller to improve the environment-robot interaction by control of both position and force. In practice, the proposed method can be used in robotic systems where constraints on joint variables are required. Robot dynamics and interaction forces, however, are not known. The ability to provide safety enables the method to obtain recent social applications besides the conventional industrial ones. Robotic surgery and the safe robotic rehabilitation are examples of recent practical applications.

\section{Appendix}

\section{Proof of Theorem 1}

Proof. (i) Uniform Boundedness (UB)

The existence of $\boldsymbol{v}_{2} \neq 0$ in (31) reveals that the system just achieves the stability, but it could not achieve the exponential stability. Based on the definition of $\boldsymbol{k}_{\boldsymbol{n} i}$ and $\boldsymbol{k}_{\boldsymbol{m} i}$ in Assumption 3, the initial condition (32) in term of initial error, $\boldsymbol{e}_{1 i}$, can be rewritten as,

$$
-\boldsymbol{k}_{n i}(0)<\boldsymbol{e}_{1 i}(0)<\boldsymbol{k}_{m i}(0)
$$

By employing Lemma 4, (A. 1) can be formed as,

$$
\left|\xi_{i}(0)\right|<1, \quad \boldsymbol{i}=1, \ldots, \boldsymbol{n}
$$

From the fact that $\dot{\boldsymbol{V}} \leq-\boldsymbol{v}_{1} \boldsymbol{V}+\boldsymbol{v}_{2}$ for all $\boldsymbol{\xi}_{\boldsymbol{i}} \in \mathbf{R}|| \boldsymbol{\xi}_{\boldsymbol{i}} \mid<1$ and using Lemma 2 and considering (A. 2), it is established that,

$$
\left|\xi_{i}\right|<1, \quad \boldsymbol{i}=1, \ldots, \boldsymbol{n}
$$

Thus, it is obtained that $-\boldsymbol{k}_{\boldsymbol{n} i}<\boldsymbol{e}_{1 i}<\boldsymbol{k}_{m i}, \boldsymbol{i}=1, \ldots, \boldsymbol{n}$, as follows from Lemma 4.

Multiplying inequality (31) by $\exp \left(\boldsymbol{v}_{1} \boldsymbol{t}\right)$ and then integrating the results leads to,

$$
0 \leq \boldsymbol{V}(\boldsymbol{t}) \leq\left(\boldsymbol{V}(0)-\frac{\boldsymbol{v}_{2}}{\boldsymbol{v}_{1}}\right) \exp \left(-\boldsymbol{v}_{1} \boldsymbol{t}\right)+\frac{\boldsymbol{\nu}_{2}}{\boldsymbol{v}_{1}} \leq \boldsymbol{V}(0)+\frac{\boldsymbol{v}_{2}}{\boldsymbol{v}_{1}}, \forall \boldsymbol{t}>0,
$$

which implies that $\boldsymbol{V}(\boldsymbol{t})$ is bounded. Accordingly, for $\boldsymbol{i}=1, \ldots, \boldsymbol{n}$, we obtain $\boldsymbol{V}(0)+\boldsymbol{v}_{2} / \boldsymbol{\nu}_{1} \geq \boldsymbol{V} \geq \boldsymbol{k}_{\boldsymbol{b i}}^{2} / \boldsymbol{\pi} \tan \left(\pi \boldsymbol{\xi}_{i}^{2} / 2\right)$. Applying some manipulations lead to $\boldsymbol{\xi}_{\boldsymbol{i}}^{2} \leq 2 \tan ^{-1}\left(\boldsymbol{\pi}\left(\boldsymbol{V}(0)+\boldsymbol{v}_{2} / \boldsymbol{v}_{1}\right) / \boldsymbol{k}_{\boldsymbol{b} i}^{2}\right) / \boldsymbol{\pi}$ which implies, 


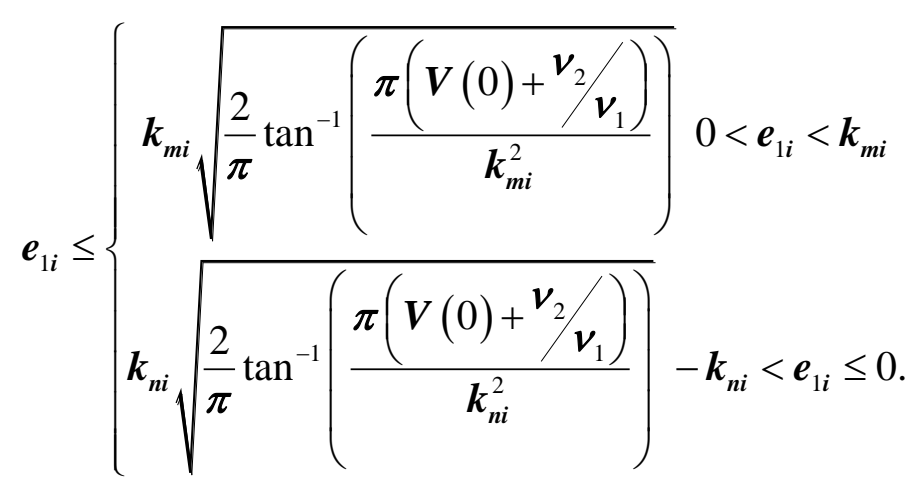

Thus, $\boldsymbol{e}_{1 i} \leq \bar{\Delta}_{i}$ for positive $\boldsymbol{e}_{1 i}$ and $\boldsymbol{e}_{1 i} \geq-\bar{\Delta}_{i}$ for negative $\boldsymbol{e}_{1 i}$. Combining both cases results in $-\underline{\Delta}_{i} \leq \boldsymbol{e}_{1 i} \leq \bar{\Delta}_{i}, \forall \boldsymbol{t}>0, \boldsymbol{i}=1, \ldots, \boldsymbol{n}$.

Combining (12) and (A.4) we have $\boldsymbol{V}(0)+\frac{\boldsymbol{v}_{2}}{\boldsymbol{v}_{1}} \geq \boldsymbol{V} \geq \frac{1}{2} \boldsymbol{e}_{2}{ }^{T} \boldsymbol{M} \boldsymbol{e}_{2} \geq \frac{1}{2} \lambda_{\min }(\boldsymbol{M})\left\|\boldsymbol{e}_{2}\right\|^{2}$ which leads to $\left\|e_{2}\right\| \leq\left(2\left(\boldsymbol{V}(0)+\boldsymbol{v}_{2} / \boldsymbol{v}_{1}\right) / \boldsymbol{\lambda}_{\min }(\boldsymbol{M})\right)^{1 / 2}$. Similarly combining (18) and (A.4) results in $\left\|\boldsymbol{e}_{3}\right\| \leq\left(2\left(\boldsymbol{V}(0)+\boldsymbol{v}_{2} / \boldsymbol{v}_{1}\right) / \boldsymbol{\lambda}_{\min }(\boldsymbol{L})\right)^{1 / 2}$.

(ii) Uniformly Ultimate Boundedness (UUB)

From (A.4) and (5), and (6), one can obtain,

$$
\boldsymbol{e}_{1 i} \leq\left\{\begin{array}{lr}
\boldsymbol{k}_{\boldsymbol{m} i} \sqrt{\frac{2}{\pi} \tan ^{-1}\left(\pi\left(\left(\boldsymbol{V}(0)-\boldsymbol{v}_{2} / \boldsymbol{v}_{1}\right) \exp \left(-\boldsymbol{v}_{1} \boldsymbol{t}\right)+\boldsymbol{v}_{2} / \boldsymbol{v}_{1}\right) / \boldsymbol{k}_{\boldsymbol{m} i}^{2}\right)} & 0<\boldsymbol{e}_{1 i}<\boldsymbol{k}_{\boldsymbol{m} i} \\
\boldsymbol{k}_{\boldsymbol{n} i} \sqrt{\frac{2}{\pi} \tan ^{-1}\left(\pi\left(\left(\boldsymbol{V}(0)-\boldsymbol{v}_{2} / \boldsymbol{v}_{1}\right) \exp \left(-\boldsymbol{v}_{1} \boldsymbol{t}\right)+\boldsymbol{v}_{2} / \boldsymbol{v}_{1}\right) / \boldsymbol{k}_{\boldsymbol{n}}^{2}\right)} & -\boldsymbol{k}_{\boldsymbol{n} i}<\boldsymbol{e}_{1 i} \leq 0 .
\end{array}\right.
$$

If $\quad \boldsymbol{V}(0)=\boldsymbol{v}_{2} / \boldsymbol{v}_{1}, \quad$ then $\quad \Lambda_{n} \leq \boldsymbol{e}_{1 i} \leq \Lambda_{m}, \quad$ with $\quad \Lambda_{n}=-\boldsymbol{k}_{n i} \sqrt{2 \tan ^{-1}\left(\pi \boldsymbol{v}_{2} / \boldsymbol{v}_{1} \boldsymbol{k}_{n i}^{2}\right) / \boldsymbol{\pi}}, \quad$ and $\Lambda_{m}=\boldsymbol{k}_{m i} \sqrt{2 \tan ^{-1}\left(\pi \boldsymbol{v}_{2} / \boldsymbol{v}_{1} \boldsymbol{k}_{m i}^{2}\right) / \pi}$. In the case that $\boldsymbol{V}(0) \neq \boldsymbol{v}_{2} / \boldsymbol{v}_{1}$, from (A.6) it is concluded that for any $\Delta_{i}>\max \left\{\underline{\Delta}_{i}^{*}, \bar{\Delta}_{i}^{*}\right\}$, there exists $\mathrm{T}_{1 i}$, such that for any $\boldsymbol{t}>\mathrm{T}_{1 i}, \quad\left|\boldsymbol{e}_{1 i}\right| \leq \Delta_{i}$. Specifically, for any $\Delta_{i}=\overline{\boldsymbol{k}}_{\boldsymbol{i}} \times \sqrt{2 \tan ^{-1}\left(\pi \Omega / \boldsymbol{k}_{i}^{2}\right) \boldsymbol{\pi}}$, where $\Omega=\left(\boldsymbol{V}(0)-\boldsymbol{v}_{2} / \boldsymbol{\nu}_{1}\right) \exp \left(-\boldsymbol{v}_{1} \mathrm{~T}_{1 i}\right)+\boldsymbol{v}_{2} / \boldsymbol{\nu}_{1}$ with $\boldsymbol{V}(0) \neq \boldsymbol{v}_{2} / \boldsymbol{v}_{1}$ and $\overline{\boldsymbol{k}}_{\boldsymbol{i}}=\max \left\{\boldsymbol{k}_{\boldsymbol{m} i}, \boldsymbol{k}_{n i}\right\}$, then,

$$
\mathrm{T}_{1 i}=-\frac{1}{\boldsymbol{v}_{1}} \ln \frac{\frac{\overline{\boldsymbol{k}}_{\boldsymbol{i}}^{2}}{\boldsymbol{\pi}} \tan \left(\frac{\pi}{2} \frac{\Delta_{i}^{2}}{\overline{\boldsymbol{k}}_{\boldsymbol{i}}^{2}}\right)-\frac{\boldsymbol{v}_{2}}{\boldsymbol{v}_{1}}}{V(0)-\frac{\boldsymbol{v}_{2}}{\boldsymbol{v}_{1}}}
$$

and 
$\lim _{\boldsymbol{t} \rightarrow \infty}\left|\boldsymbol{e}_{1 i}(\boldsymbol{t})\right|=\max \left\{\underline{\Delta}_{i}^{*}, \bar{\Delta}_{i}^{*}\right\}$

Following a procedure similar to that in $\boldsymbol{e}_{1}$, one can obtain,

$$
\begin{aligned}
& \left\|e_{2}\right\| \leq \sqrt{\frac{2\left(\boldsymbol{V}(0)-\frac{\boldsymbol{v}_{2}}{\boldsymbol{v}_{1}}\right) \exp \left(-\boldsymbol{v}_{1} \boldsymbol{t}\right)+\frac{2 \boldsymbol{v}_{2}}{\boldsymbol{v}_{1}}}{\lambda_{\min }(M)},} \\
& \left\|\boldsymbol{e}_{3}\right\| \leq \sqrt{\frac{2\left(\boldsymbol{V}(0)-\frac{\boldsymbol{v}_{2}}{\boldsymbol{v}_{1}}\right) \exp \left(-\boldsymbol{v}_{1} \boldsymbol{t}\right)+\frac{2 \boldsymbol{v}_{2}}{\boldsymbol{v}_{1}}}{\lambda_{\min }(L)}} .
\end{aligned}
$$

Then, with $\boldsymbol{V}(0)=\boldsymbol{v}_{2} / \boldsymbol{v}_{1}, \quad\left\|\boldsymbol{e}_{2}\right\| \leq \sqrt{2 \boldsymbol{v}_{2} / \boldsymbol{v}_{1} \lambda_{\min }(\boldsymbol{M})}=\boldsymbol{\varepsilon}^{*}{ }_{2}$ and $\left\|\boldsymbol{e}_{3}\right\| \leq \sqrt{2 \boldsymbol{v}_{2} / \boldsymbol{v}_{1} \lambda_{\min }(\boldsymbol{L})}=\boldsymbol{\varepsilon}^{*}{ }_{3}$; and if $\boldsymbol{V}(0) \neq \boldsymbol{v}_{2} / \boldsymbol{v}_{1}$, from (A.9) it is concluded that given any $\boldsymbol{\varepsilon}_{2}>\boldsymbol{\varepsilon}_{2}{ }_{2}$ and $\boldsymbol{\varepsilon}_{3}>\boldsymbol{\varepsilon}_{3}{ }_{3}$, there exists $\mathrm{T}_{2}$ and $\mathrm{T}_{3}$, such that for any $\boldsymbol{t}>\mathrm{T}_{2 i}$ and $\boldsymbol{t}>\mathrm{T}_{3 i}$ we have $\left\|\boldsymbol{e}_{2}\right\| \leq \boldsymbol{\varepsilon}_{2}$ and $\left\|\boldsymbol{e}_{3}\right\| \leq \boldsymbol{\varepsilon}_{3}$, respectively. Specifically, given any $\varepsilon_{2}$ and $\varepsilon_{3}$ as,

$$
\begin{aligned}
& \boldsymbol{\varepsilon}_{2}=\sqrt{\frac{2\left(\boldsymbol{V}(0)-\frac{\boldsymbol{v}_{2}}{\boldsymbol{v}_{1}}\right) \exp \left(-\boldsymbol{v}_{1} \mathrm{~T}_{2}\right)+\frac{2 \boldsymbol{v}_{2}}{\boldsymbol{v}_{1}}}{\lambda_{\min }(\boldsymbol{M})},} \quad \boldsymbol{V}(0) \neq \boldsymbol{v}_{2} / \boldsymbol{v}_{1}, \\
& \boldsymbol{\varepsilon}_{3}=\sqrt{\frac{2\left(\boldsymbol{V}(0)-\frac{\boldsymbol{v}_{2}}{\boldsymbol{v}_{1}}\right) \exp \left(-\boldsymbol{v}_{1} \mathrm{~T}_{3}\right)+\frac{2 \boldsymbol{v}_{2}}{\boldsymbol{v}_{1}}}{\lambda_{\min }(\boldsymbol{L})},} \boldsymbol{V}(0) \neq \boldsymbol{v}_{2} / \boldsymbol{v}_{1},
\end{aligned}
$$

then,

$$
\begin{gathered}
\mathrm{T}_{2}=-\frac{1}{\boldsymbol{v}_{1}} \ln \left(\frac{\boldsymbol{\varepsilon}_{2}{ }^{2} \lambda_{\min }(\boldsymbol{M})-\frac{2 \boldsymbol{v}_{2}}{\boldsymbol{v}_{1}}}{2\left(\boldsymbol{V}(0)-\frac{\boldsymbol{v}_{2}}{\boldsymbol{v}_{1}}\right)}\right), \\
\mathrm{T}_{3}=-\frac{1}{\boldsymbol{v}_{1}} \ln \left(\frac{\boldsymbol{\varepsilon}_{3}^{2} \lambda_{\min }(\boldsymbol{L})-\frac{2 \boldsymbol{v}_{2}}{\boldsymbol{v}_{1}}}{2\left(\boldsymbol{V}(0)-\frac{\boldsymbol{v}_{2}}{\boldsymbol{v}_{1}}\right)}\right) .
\end{gathered}
$$

and

$$
\begin{aligned}
& \lim _{\boldsymbol{t} \rightarrow \infty}\left\|\boldsymbol{e}_{2}(\boldsymbol{t})\right\|=\boldsymbol{\varepsilon}^{*}{ }_{2}, \\
& \lim _{\boldsymbol{t} \rightarrow \infty}\left\|\boldsymbol{e}_{3}(\boldsymbol{t})\right\|=\boldsymbol{\varepsilon}^{*}{ }_{3} .
\end{aligned}
$$


(iii) From $\boldsymbol{q}_{1 i}=\boldsymbol{x}_{1 i}=\boldsymbol{e}_{1 i}+\boldsymbol{x}_{d i}, \quad-\underline{\boldsymbol{k}}_{d i}<\boldsymbol{x}_{d i}<\overline{\boldsymbol{k}}_{d i}$, and $-\underline{\Delta}_{i} \leq \boldsymbol{e}_{1 i} \leq \bar{\Delta}_{i}$, it can be concluded that $-\underline{\Delta}_{i}-\underline{\boldsymbol{k}}_{d i} \leq \boldsymbol{q}_{i} \leq \bar{\Delta}_{i}+\overline{\boldsymbol{k}}_{d i}$. Then, since $\bar{\Delta}_{i} \leq \boldsymbol{k}_{\boldsymbol{m} i}$ it can be shown that $\bar{\Delta}_{i}+\overline{\boldsymbol{k}}_{d i} \leq \boldsymbol{k}_{\boldsymbol{m} i}+\overline{\boldsymbol{k}}_{d i} \leq \overline{\boldsymbol{k}}_{\boldsymbol{o} i}$. Similarly, since $\underline{\Delta}_{i} \leq \boldsymbol{k}_{n i}$, then $\underline{\Delta}_{i}+\underline{\boldsymbol{k}}_{d i} \leq \boldsymbol{k}_{\boldsymbol{n} i}+\underline{\boldsymbol{k}}_{d i} \leq \underline{\boldsymbol{k}}_{o i}$. Thus, we can conclude that $\boldsymbol{q}_{i} \in \Omega_{y}$.

(iv) Signals $\boldsymbol{e}_{1}, \boldsymbol{e}_{2}, \boldsymbol{e}_{3}$ and $\boldsymbol{q}_{1}$ are bounded, as shown in (i) and (iii). From Assumptions 2, and 3, it can be concluded that $\dot{\boldsymbol{k}}_{\boldsymbol{m} i}$ and $\dot{\boldsymbol{k}}_{\boldsymbol{n} i}$ are bounded with the estimated bound as $\left|\dot{\boldsymbol{k}}_{\boldsymbol{m} i}\right| \leq \boldsymbol{X}_{\boldsymbol{d 1 i}}+\overline{\boldsymbol{K}}_{\boldsymbol{o} \boldsymbol{i}}$ and $\left|\dot{\boldsymbol{k}}_{n i}\right| \leq \boldsymbol{X}_{\boldsymbol{d} 1 i}+\underline{\boldsymbol{K}}_{\boldsymbol{i} i}$. Thus, it is clear, from Assumption 2 that the stabilizing function $\boldsymbol{\alpha}$ is also bounded. This leads to boundedness of $\boldsymbol{x}_{2}$ as $\boldsymbol{x}_{2}=\boldsymbol{e}_{2}+\boldsymbol{\alpha}$. Since $\boldsymbol{V}(\boldsymbol{t}) \leq \boldsymbol{V}(0)+\boldsymbol{v}_{2} / \boldsymbol{v}_{1}, \forall \boldsymbol{t}>0$, then $\hat{W}_{1}, \hat{W}_{2}, \hat{D}_{r}$ and $\hat{D}_{a}$ are all bounded. Also, as $\boldsymbol{h}_{1}$ and $f$ are bounded, it is clear from (16) and (17) that $\tau_{d}$ is bounded in the set $\left|\xi_{i}\right|<1$. Thus, from Lemma 4 and Assumption 3 one can conclude that $\tau_{d}$ is bounded within $\Omega_{y}$. This leads to the boundedness of $\boldsymbol{x}_{3}$, since $\boldsymbol{x}_{3}=\boldsymbol{e}_{3}+\tau_{d}$. Finally, from bounding $\boldsymbol{u}$ as $\hat{\boldsymbol{W}}_{2}$ and $\hat{\boldsymbol{D}}_{\boldsymbol{a}}$, and $\boldsymbol{h}_{2}$ are bounded, we conclude that all closed loop signals are bounded.

\section{References}

[1] W. He, W. Ge, Y. Li, Y.-J. Liu, C. Yang, C. Sun, Model identification and control design for a humanoid robot, IEEE Transactions on Systems, Man, and Cybernetics: Systems, 47 (2017) 45 - 57.

[2] Z. Liu, C. Chen, Y. Zhang, C. Chen, Adaptive neural control for dual-arm coordination of humanoid robot with unknown nonlinearities in output mechanism, IEEE Transactions on Cybernetics, 45 (2015) 521-532.

[3] B.V. Adorno, A.P.L. Bo, P. Fraisse, Kinematic modeling and control for human-robot cooperation considering different interaction roles, Robotica, 33 (2015) 314-331.

[4] W. Gallagher, M. Ding, J. Ueda, Relaxed individual control of skeletal muscle forces via physical human-robot interaction, Multibody System Dynamics, 30 (2013) 77-99.

[5] P. Maciejasz, J. Eschweiler, K. Gerlach-Hahn, A. Jansen-Troy, S. Leonhardt, A survey on robotic devices for upper limb rehabilitation, Journal of neuroengineering and rehabilitation, 11 (2014) 1.

[6] Z. Li, S.S. Ge, A. Ming, Adaptive robust motion/force control of holonomic-constrained nonholonomic mobile manipulators, IEEE Transactions on Systems, Man, and Cybernetics, Part B: Cybernetics, 37 (2007) 607-616.

[7] C.C. Cheah, S.P. Hou, Y. Zhao, J.-J.E. Slotine, Adaptive vision and force tracking control for robots with constraint uncertainty, IEEE/ASME Transactions on Mechatronics, 15 (2010) 389-399.

[8] Y. Li, S.S. Ge, C. Yang, Learning impedance control for physical robot-environment interaction, International Journal of Control, 85 (2012) 182-193.

[9] B. Lacevic, P. Rocco, A.M. Zanchettin, Safety assessment and control of robotic manipulators using danger field, IEEE Transactions on Robotics, 29 (2013) 1257-1270.

[10] G. Buizza Avanzini, N.M. Ceriani, A.M. Zanchettin, P. Rocco, L. Bascetta, Safety control of industrial robots based on a distributed distance sensor, IEEE Transactions on Control Systems Technology, 22 (2014) 2127-2140.

[11] B. Fan, Q. Yang, S. Jagannathan, Y. Sun, Asymptotic Tracking Controller Design for Nonlinear Systems With Guaranteed Performance, IEEE transactions on cybernetics, (2017) DOI: 10.1109/TCYB.2017.2726039.

[12] M.H. Korayem, H. Ghariblu, Maximum allowable load on wheeled mobile manipulators imposing redundancy constraints, Robotics and Autonomous Systems, 44 (2003) 151-159.

[13] M.H. Korayem, H. Ghariblu, Analysis of wheeled mobile flexible manipulator dynamic motions with maximum load carrying capacities, Robotics and Autonomous Systems, 48 (2004) 63-76.

[14] O. Khatib, Real-time obstacle avoidance for manipulators and mobile robots, The international journal of robotics research, 5 (1986) 90-98.

[15] Z. Zhang, Y. Zhang, Variable joint-velocity limits of redundant robot manipulators handled by quadratic programming, IEEE/ASME Transactions on Mechatronics, 18 (2013) 674-686.

[16] M.H. Korayem, M. Nazemizadeh, H.R. Nohooji, Optimal point-to-point motion planning of non-holonomic mobile robots in the presence of multiple obstacles, Journal of the Brazilian Society of Mechanical Sciences and Engineering, 36 (2014) 221232. 
[17] M. Korayem, M. Nazemizadeh, H. Rahimi, Trajectory optimization of nonholonomic mobile manipulators departing to a moving target amidst moving obstacles, Acta Mechanica, 224 (2013) 995-1008.

[18] K.B. Ngo, R. Mahony, Z.-P. Jiang, Integrator backstepping design for motion systems with velocity constraint, 5th Asian Control Conference, Melbourne, Victoria, Australia, 2004.

[19] K.B. Ngo, R. Mahony, Z.-P. Jiang, Integrator backstepping using barrier functions for systems with multiple state constraints, 44th IEEE Conference on Decision and Control, 2005 and 2005 European Control Conference. CDC-ECC'05, IEEE, 2005, pp. 8306-8312.

[20] K.P. Tee, S.S. Ge, E.H. Tay, Barrier Lyapunov functions for the control of output-constrained nonlinear systems, Automatica, 45 (2009) 918-927.

[21] B. Ren, S.S. Ge, K.P. Tee, T.H. Lee, Adaptive neural control for output feedback nonlinear systems using a barrier Lyapunov function, IEEE Transactions on Neural Networks, 21 (2010) 1339-1345.

[22] Y.-J. Liu, S. Tong, Barrier Lyapunov functions-based adaptive control for a class of nonlinear pure-feedback systems with full state constraints, Automatica, 64 (2016) 70-75.

[23] Z.-L. Tang, S.S. Ge, K.P. Tee, W. He, Adaptive neural control for an uncertain robotic manipulator with joint space constraints, International Journal of Control, 89 (2016) 1428-1446.

[24] Z.-L. Tang, K.P. Tee, W. He, Tangent barrier Lyapunov functions for the control of output-constrained nonlinear systems, IFAC Proceedings Volumes, 46 (2013) 449-455.

[25] X. Jin, Adaptive fault tolerant control for a class of input and state constrained MIMO nonlinear systems, International Journal of Robust and Nonlinear Control, 26 (2016) 286-302.

[26] H.N. Rahimi, I. Howard, L. Cui, Neural Network Adaptive Control Design for Robot Manipulators under Velocity Constraints, Journal of the Franklin Institute, 355 (2018) 693-713.

[27] Z. Chen, Z. Li, C.P. Chen, Adaptive neural control of uncertain MIMO nonlinear systems with state and input constraints, IEEE transactions on neural networks and learning systems, 28 (2017) 1318-1330.

[28] Y. Qiu, Z. Dai, Y. Chen, X. Liang, Constrained Control for Brushless DC Motors With Fractional Friction Compensation, ASME International Design Engineering Technical Conferences and Computers and Information in Engineering Conference, American Society of Mechanical Engineers, Boston, Massachusetts, 2015.

[29] S. Luo, J. Wang, S. Wu, K. Xiao, Chaos RBF dynamics surface control of brushless DC motor with time delay based on tangent barrier Lyapunov function, Nonlinear Dynamics, 78 (2014) 1193-1204.

[30] H. Habibi, H.N. Rahimi, I. Howard, Constrained Control of Wind Turbines for Power Regulation in Full Load Operation, 11 th Asian Control Conference, Gold Coast, Australia, 2017, pp. 2813-2818.

[31] W. He, S. Zhang, S.S. Ge, Adaptive control of a flexible crane system with the boundary output constraint, IEEE Transactions on Industrial Electronics, 61 (2014) 4126-4133.

[32] W. He, S.S. Ge, Vibration control of a flexible string with both boundary input and output constraints, IEEE Transaction s on Control Systems Technology, 23 (2015) 1245-1254.

[33] W. He, S.S. Ge, Vibration control of a flexible beam with output constraint, IEEE Transactions on Industrial Electronics, 62 (2015) 5023-5030.

[34] W. He, C. Sun, S.S. Ge, Top tension control of a flexible marine riser by using integral-barrier Lyapunov function, IEEE/ASME Transactions on Mechatronics, 20 (2015) 497-505.

[35] Z. Zuo, C. Wang, Adaptive trajectory tracking control of output constrained multi-rotors systems, Control Theory \& Applications, IET, 8 (2014) 1163-1174.

[36] H. An, H. Xia, C. Wang, Barrier Lyapunov function-based adaptive control for hypersonic flight vehicles, Nonlinear Dynamics, 88 (2017) 1833-1853.

[37] L. Sun, W. Huo, Z. Jiao, Adaptive Backstepping Control of Spacecraft Rendezvous and Proximity Operations With Input Saturation and Full-State Constraint, IEEE Transaction on Industrial Electronics, 64 (2017) 480-492.

[38] Z. Zheng, Y. Huang, L. Xie, B. Zhu, Adaptive trajectory tracking control of a fully actuated surface vessel with asymmetrically constrained input and output, IEEE Transactions on Control Systems Technology, Inpress (2017) DOI: 10.1109/TCST.2017.2728518.

[39] J. Ghommam, M. Saad, Adaptive Leader-Follower Formation Control of Underactuated Surface Vessels Under Asymmetric Range and Bearing Constraints, IEEE Transactions on Vehicular Technology, 67 (2018) 852 - 865.

[40] K.P. Tee, R. Yan, H. Li, Adaptive admittance control of a robot manipulator under task space constraint, Robotics and Automation (ICRA), 2010 IEEE International Conference on, IEEE, 2010, pp. 5181-5186.

[41] K.P. Tee, S.S. Ge, R. Yan, H. Li, Adaptive control for robot manipulators under ellipsoidal task space constraints, Intelligent Robots and Systems (IROS), 2012 IEEE/RSJ International Conference on, IEEE, 2012, pp. 1167-1172.

[42] C.-C. Cheah, C. Liu, J. Slotine, Adaptive Jacobian tracking control of robots with uncertainties in kinematic, dynamic and actuator models, IEEE Transactions on Automatic Control, 51 (2006) 1024-1029.

[43] W. He, A.O. David, Z. Yin, C. Sun, Neural network control of a robotic manipulator with input deadzone and output constraint, IEEE Transactions on Systems, Man, and Cybernetics: Systems, 46 (2016) 759-770.

[44] X. Jin, J.-X. Xu, A barrier composite energy function approach for robotmanipulators under alignment condition with position constraints, International Journal of Robust and Nonlinear Control, 24 (2014) 2840-2851. 
[45] W. He, Y. Chen, Z. Yin, Adaptive neural network control of an uncertain robot with full-state constraints, IEEE Transactions on Cybernetics, 46 (2016) 620-629.

[46] C. Wen, J. Zhou, Z. Liu, H. Su, Robust adaptive control of uncertain nonlinear systems in the presence of input saturation and external disturbance, IEEE Transactions on Automatic Control, 56 (2011) 1672-1678.

[47] Z.-L. Tang, S.S. Ge, W. He, Neural adaptive control for robots with uncertainties in manipulator dynamics and actuator dynamics under constrained task space, 34th Chinese Control Conference, IEEE, 2015, pp. 5824-5829.

[48] J. Park, I.W. Sandberg, Universal approximation using radial-basis-function networks, Neural computation, 3 (1991) 246257.

[49] L.-X. Wang, Adaptive fuzzy systems and control: design and stability analysis, Prentice-Hall, Inc., 1994.

[50] H. Habibi, H.N. Rahimi, I. Howard, Optimum efficiency control of a wind turbine with unknown desired trajectory and actuator faults, Journal of Renewable and Sustainable Energy, 9 (2017) 063305.

[51] L. Liu, Y.-J. Liu, C.P. Chen, Adaptive neural network control for a DC motor system with dead-zone, Nonlinear Dynamics, 72 (2013) 141-147.

[52] J. Guo, Y. Luo, K. Li, Adaptive neural-network sliding mode cascade architecture of longitudinal tracking control for unmanned vehicles, Nonlinear Dynamics, 87 (2017) 2497-2510.

[53] H.F. Ghavidel, A.A. Kalat, Robust control for MIMO hybrid dynamical system of underwater vehicles by composite adaptive fuzzy estimation of uncertainties, Nonlinear Dynamics, (2017) 1-19.

[54] N. Wang, M.J. Er, Direct adaptive fuzzy tracking control of marine vehicles with fully unknown parametric dynamics and uncertainties, IEEE Transactions on Control Systems Technology, 24 (2016) 1845-1852.

[55] H. Rahimi, M. Nazemizadeh, Dynamic analysis and intelligent control techniques for flexible manipulators: a review, Advanced Robotics, 28 (2013) 63-76.

[56] C. Yang, Z. Li, J. Li, Trajectory planning and optimized adaptive control for a class of wheeled inverted pendulum vehicle models, IEEE Transactions on Cybernetics, 43 (2013) 24-36.

[57] Z. Li, C.-Y. Su, L. Wang, Z. Chen, T. Chai, Nonlinear disturbance observer-based control design for a robotic exoskeleton incorporating fuzzy approximation, IEEE Transactions on Industrial Electronics, 62 (2015) 5763-5775.

[58] Y.-J. Liu, S. Tong, C.P. Chen, D.-J. Li, Neural controller design-based adaptive control for nonlinear MIMO systems with unknown hysteresis inputs, IEEE transactions on cybernetics, 46 (2016) 9-19.

[59] D.S. Broomhead, D. Lowe, Radial basis functions, multi-variable functional interpolation and adaptive networks, DTIC Document, 1988.

[60] J. Liu, Radial Basis Function (RBF) neural network control for mechanical systems: design, analysis and Matlab simulation, Springer Science \& Business Media, 2013.

[61] S. Yang, Y. Cao, Z. Peng, G. Wen, K. Guo, Distributed formation control of nonholonomic autonomous vehicle via RBF neural network, Mechanical Systems and Signal Processing, 87 (2017) 81-95.

[62] H.N. Rahimi, I. Howard, L. Cui, Neural Adaptive Assist-As-Needed Control for Rehabilitation Robots, Australian Conference on Robotics and Automation (ACRA), Brisbane, Australia, 2016.

[63] M. Rahmani, A. Ghanbari, M.M. Ettefagh, Hybrid neural network fraction integral terminal sliding mode control of an Inchworm robot manipulator, Mechanical Systems and Signal Processing, 80 (2016) 117-136.

[64] H. Rahimi N., I. Howard, L. Cui, Neural impedance adaption for assistive human-robot interaction, Neurocomputing, (2018) in press.

[65] X. Jin, Adaptive fault-tolerant control for a class of output-constrained nonlinear systems, International Journal of Robust and Nonlinear Control, 25 (2015) 3732-3745.

[66] X. Jin, Adaptive fault tolerant tracking control for a class of stochastic nonlinear systems with output constraint and actuator faults, Systems \& Control Letters, 107 (2017) 100-109.

[67] X. Jin, Adaptive decentralized finite-time output tracking control for MIMO interconnected nonlinear systems with output constraints and actuator faults, International Journal of Robust and Nonlinear Control, 28 (2018) 1808-1829.

[68] T. Li, S. Duan, J. Liu, L. Wang, T. Huang, A Spintronic Memristor-Based Neural Network With Radial Basis Function for Robotic Manipulator Control Implementation, IEEE Transactions on Systems, Man, and Cybernetics: Systems, 46 (2016) 582 588.

[69] W. He, Y. Dong, C. Sun, Adaptive neural impedance control of a robotic manipulator with input saturation, IEEE Transactions on Systems, Man, and Cybernetics: Systems, 46 (2015) 334 - 344.

[70] T. Guo, X. Wu, Backstepping control for output-constrained nonlinear systems based on nonlinear mapping, Neural Computing and Applications, 25 (2014) 1665-1674.

[71] L. Liu, Z. Wang, H. Zhang, Adaptive dynamic surface error constrained control for MIMO systems with backlash-like hysteresis via prediction error technique, Nonlinear Dynamics, 84 (2016) 1989-2002.

[72] W. Meng, Q. Yang, Y. Sun, Adaptive neural control of nonlinear MIMO systems with time-varying output constraints, IEEE Transactions on Neural Networks and Learning Systems, 26 (2015) 1074-1085.

[73] W. Meng, Q. Yang, J. Si, Y. Sun, Adaptive Neural Control of a Class of Output-Constrained Nonaffine Systems, IEEE Transactions on Cybernetics, 46 (2016) 85-95.

[74] F. Lewis, S. Jagannathan, A. Yesildirak, Neural network control of robot manipulators and non-linear systems, CRC Press, 1998. 
[75] J.-J.E. Slotine, W. Li, On the adaptive control of robot manipulators, The International Journal of Robotics Research, 6 (1987) 49-59.

[76] T.-J. Tarn, A.K. Bejczy, X. Yun, Z. Li, Effect of motor dynamics on nonlinear feedback robot arm control, IEEE Transactions on Robotics and Automation, 7 (1991) 114-122.

[77] K.P. Tee, B. Ren, S.S. Ge, Control of nonlinear systems with time-varying output constraints, Automatica, 47 (2011) 2511-2516.

[78] M.M. Polycarpou, P.A. Ioannou, A robust adaptive nonlinear control design, Automatica, 32 (1996) $423-427$.

[79] X. Jin, Fault tolerant finite-time leader-follower formation control for autonomous surface vessels with LOS range and angle constraints, Automatica, 68 (2016) 228-236.

[80] J.-J.E. Slotine, W. Li, Applied nonlinear control, Prentice-Hall Englewood Cliffs, NJ, 1991.

[81] S.S. Ge, C. Wang, Adaptive neural control of uncertain MIMO nonlinear systems, IEEE Transactions on Neural Networks, 15 (2004) 674-692.

[82] D.-H. Zhai, Y. Xia, Adaptive control for teleoperation system with varying time-delays and input saturation constraints, IEEE Transactions on Industrial Electronics 63 (2016) 6921 - 6929.

[83] S. Gao, B. Ning, H. Dong, Fuzzy dynamic surface control for uncertain nonlinear systems under input saturation via truncated adaptation approach, Fuzzy Sets and Systems, 290 (2016) 100-117.

[84] W. He, X. He, S.S. Ge, Vibration control of flexible marine riser systems with input saturation, IEEE/ASME Transactions on Mechatronics, 21 (2016) 254-265.

[85] Y. Li, S. Tong, T. Li, Composite adaptive fuzzy output feedback control design for uncertain nonlinear strict-feedback systems with input saturation, IEEE Transactions on Cybernetics, 45 (2015) 2299-2308.

[86] Z.-L. Tang, S.S. Ge, K.P. Tee, W. He, Robust adaptive neural tracking control for a class of perturbed uncertain nonlinear systems with state constraints, IEEE Transactions on Systems, Man, and Cybernetics: Systems 46 (2016) 1618 - 1629.

[87] K. Zhao, Y. Song, Z. Shen, Neuroadaptive fault-tolerant control of nonlinear systems under output constraints and actuation faults, IEEE transactions on neural networks and learning systems, (2016).

[88] Y. Liu, J. Yu, H. Yu, C. Lin, L. Zhao, Barrier Lyapunov functions-based adaptive neural control for permanent magnet synchronous motors with full-state constraints, IEEE Access, 5 (2017) 10382 - 10389.

[89] Z.-J. Jia, Y.-D. Song, Barrier Function-Based Neural Adaptive Control With Locally Weighted Learning and Finite Neuron Self-Growing Strategy, IEEE Transactions on Neural Networks and Learning Systems, 28 (2017) 1439-1451. 